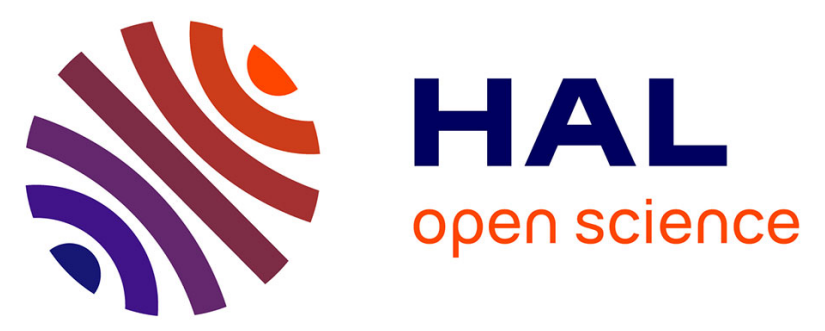

\title{
The DREAMS Experiment Onboard the Schiaparelli Module of the ExoMars 2016 Mission: Design, Performances and Expected Results
}

Francesca Esposito, Stefano Debei, C. Bettanini, C. Molfese, I. Arruego Rodríguez, G. Colombatti, A.-M. Harri, Franck Montmessin, C. Wilson, A. Aboudan, et al.

\section{To cite this version:}

Francesca Esposito, Stefano Debei, C. Bettanini, C. Molfese, I. Arruego Rodríguez, et al.. The DREAMS Experiment Onboard the Schiaparelli Module of the ExoMars 2016 Mission: Design, Performances and Expected Results. Space Science Reviews, 2018, 214 (6), pp.art. 103. 10.1007/s11214018-0535-0 . insu-01858965

\section{HAL Id: insu-01858965 \\ https://hal-insu.archives-ouvertes.fr/insu-01858965}

Submitted on 26 Nov 2020

HAL is a multi-disciplinary open access archive for the deposit and dissemination of scientific research documents, whether they are published or not. The documents may come from teaching and research institutions in France or abroad, or from public or private research centers.
L'archive ouverte pluridisciplinaire HAL, est destinée au dépôt et à la diffusion de documents scientifiques de niveau recherche, publiés ou non, émanant des établissements d'enseignement et de recherche français ou étrangers, des laboratoires publics ou privés. 


\section{Authors}

F. Esposito ${ }^{1}$, S. Debei ${ }^{2}$, C. Bettanini' ${ }^{2}$, C. Molfese ${ }^{1}$, I. Arruego Rodríguez ${ }^{3}$, G. Colombatti' ${ }^{2}$, A-M. Harri ${ }^{4}$, F. Montmessin $^{5}$, C. Wilson ${ }^{6}$, A. Aboudan' ${ }^{2}$, P. Schipani ${ }^{1}$, L. Marty ${ }^{1}$, F.J. Álvarez ${ }^{3}$, V. Apestigue ${ }^{3}$, G. Bellucci ${ }^{7}, J^{-J}$. Berthelier $^{5}$, J. R. Brucato ${ }^{8}$, S. B. Calcutt ${ }^{6}$, S. Chiodini ${ }^{2}$, F. Cortecchia ${ }^{20}$, F. Cozzolino ${ }^{1}$, F. Cucciarrè2 , N. Deniskina $^{1}$, G. Déprez ${ }^{5}$, G. Di Achille ${ }^{24}$, F. Ferri ${ }^{2}$, F. Forget ${ }^{9}$, G. Franzese ${ }^{1,10}$, E. Friso ${ }^{2}$, M. Genzer ${ }^{4}$, R. HassenKodja $^{5}$, H. Haukka ${ }^{4}$, M. Hieta ${ }^{4}$, J. J. Jiménez ${ }^{3}$, J-L. Josset ${ }^{11}$, H. Kahanpää ${ }^{4}$, O. Karatekin ${ }^{12}$, G. Landis ${ }^{13}$, L. Lapauw $^{5}$, R. Lorenz ${ }^{14}$, J. Martinez-Oter ${ }^{3}$, V. Mennella ${ }^{1}$, D. Möhlmann ${ }^{15}$, D. Moirin ${ }^{5}$, R. Molinaro ${ }^{1}$, T. Nikkanen ${ }^{4}$, E. Palomba ${ }^{7}$, M.R. Patel ${ }^{16}$, J-P. Pommereau ${ }^{5}$, C.I. Popa ${ }^{1}$, S. Rafkin ${ }^{17}$, P. Rannou ${ }^{18}$, N.O. Renno ${ }^{19}$, J. Rivas ${ }^{3}$, W. Schmidt $^{4}$, E. Segato ${ }^{2}$, S. Silvestro ${ }^{1}$, A. Spiga ${ }^{9}$, D. Toledo ${ }^{25}$, R. Trautner ${ }^{22}$, F. Valero ${ }^{21}$, L. Vázquez ${ }^{21}$, F. Vivat ${ }^{5}$, 0. Witasse $^{22}$, M. Yela ${ }^{3}$, R. Mugnuolo ${ }^{23}$, E. Marchetti ${ }^{23}$, S. Pirrotta ${ }^{23}$

\section{Affiliations}

${ }^{1} I N A F$ - Osservatorio Astronomico di Capodimonte, Napoli, Italy, ${ }^{2}$ CISAS - Università degli Studi di Padova, Padova, Italy, ${ }^{3}$ INTA, Madrid, Spain, ${ }^{4}$ Finnish Meteorological Institute (FMI),Helsinki, Finland, ${ }^{5}$ LATMOS - CNRS/UVSQ/IPSL, France, ${ }^{6}$ Oxford University, Oxford, United Kingdom, ${ }^{7}$ INAF - Istituto di Fisica dello Spazio Interplanetario (IFSI), ${ }^{8}$ INAF-Osservatorio Astrofisico di Arcetri, ${ }^{9}$ Laboratoire de Météorologie Dynamique, UMR CNRS 8539, Institut Pierre-Simon Laplace, Sorbonne Universités, UPMC Univ Paris 06, Centre National de la Recherche Scientifique, Paris, France, ${ }^{10}$ Department of physics - University of Naples "Federico II", Naples, Italy,, ${ }^{11}$ Space Exploration Institute, Switzerland, ${ }^{12}$ Royal Observatory of Belgium,Belgium, ${ }^{13}$ NASA, GRC, USA, ${ }^{14} \mathrm{JHU}$ Applied Physics Lab (JHUAPL), USA, ${ }^{15}$ DLR PF Leitungsbereich, Berlin, Germany, ${ }^{16}$ Open University, UK, ${ }^{17}$ Southwest Research Institute, USA, ${ }^{18} \mathrm{GSMA}$, France, ${ }^{19}$ University of Michigan, USA, ${ }^{20} \mathrm{INAF}$ - Osservatorio Astronomico di Bologna, Bologna, Italy, ${ }^{21}$ Universidad Complutense de Madrid (UCM), Spain, ${ }^{22}$ ESA-ESTEC, Noordwijk, The Netherlands, ${ }^{23}$ Italian Space Agency, Italy, ${ }^{24}$ INAF - Osservatorio Astronomico di Teramo, Teramo, Italy, ${ }^{25}$ Reims Champagne-Ardenne University, France.

Corresponding author:

Francesca Esposito

INAF - Osservatorio Astronomico di Capodimonte

Salita Moiariello 16, 80131 Naples, Italy

Email: francesca.esposito@na.astro.it

Key points 
The first of the two missions foreseen in the ExoMars program was successfully launched on $14^{\text {th }}$

40

41

42

43

March 2016. It included the Trace Gas Orbiter and the Schiaparelli Entry descent and landing

Demonstrator Module. Schiaparelli hosted the DREAMS instrument suite that was the only scientific payload designed to operate after the touchdown. DREAMS is a meteorological station with the capability of measuring the electric properties of the Martian atmosphere. It was a completely autonomous instrument, relying on its internal battery for the power supply. Even with low resources (mass, energy), DREAMS would be able to perform novel measurements on Mars (atmospheric electric field) and further our understanding of the Martian environment, including the dust cycle. DREAMS sensors were designed to operate in a very dusty environment, because it was designed to land on Mars during the dust storm season (October 2016 in Meridiani Planum).

Unfortunately, the Schiaparelli module failed part of the descent and the landing and crashed onto the surface of Mars. Nevertheless, several seconds before the crash, the module central computer switched the DREAMS instrument on, and sent back housekeeping data indicating that the DREAMS sensors were performing nominally. This article describes the instrument in terms of scientific goals, design, working principle and performances, as well as the results of calibration and field tests. The spare model is mature and available to fly in a future mission. 


\section{Introduction}

The European Space Agency (ESA) has been executing the ExoMars program in cooperation with the Russian federal Space Agency (Roscosmos). It foresees two elements: the first one, launched on $14^{\text {th }}$ March 2016 from Baikonur in Kazakhstan, includes the Trace Gas Orbiter (TGO), currently in orbit around Mars, and the Schiaparelli lander demonstrator that unfortunately did not land safely. The second mission will be launched in the 2020; it includes a Russian Surface Platform and a European rover. The whole program will allow Europe to mature the technologies necessary for the entry, descent and landing of a payload on the surface of Mars, to move on the Martian surface with a rover, to penetrate into the subsurface and acquire samples and to distribute the collected samples to on-board instruments for analysis. From the scientific point of view, the ExoMars program will search for signs of extant or extinct life forms, will monitor trace gases in the atmosphere of Mars and their sources, will investigate how the water and geochemical environment varies, will monitor long-term climate and will perform atmospheric investigations (Vago et al., 2015).

The 2016 mission arrived at Mars on $19^{\text {th }}$ October 2016. The TGO successfully entered into Martian orbit and started the acquisition of scientific data around the end of November. Its main scientific objectives are to monitor methane and other trace gases and their sources on the surface of Mars. Since living organisms produce most of the methane present in the terrestrial atmosphere, the science community has interest in the monitoring of this gas on Mars.

While TGO successfully entered into Martian orbit on $19^{\text {th }}$ October, unfortunately the Schiaparelli module failed to land safely. This module carried the DREAMS (Dust characterization, Risk assessment and Environment Analyzer on the Martian Surface) instrument, that was supposed to start to operate after the touchdown in Meridiani Planum, an active area from the aeolian point of view (Chojnacki et al., 2016; Silvestro et al., 2011; 2015). A few seconds before crashing onto the surface of Mars, Schiaparelli started the sequence of operations foreseen after landing and switched on DREAMS that proved to be healthy and ready to start measurements. 
DREAMS is a meteorological station, conceived with a modular architecture, with the capability of performing measurements of the electric field close to the surface of Mars. It is an autonomous system that includes its own battery to supply power. It includes the following subsystems (seeSection 2): MarsTEM (thermometer), DREAMS-P (pressure sensor), DREAMS-H (humidity sensor), MetWind (2-D wind sensor), MicroARES (electric field sensor), SIS (Solar Irradiance Sensor), a CEU (Central Electronic Unit) and a battery. It was designed to operate in very extreme conditions such as during the dust storm season, when it was supposed to land.

DREAMS is mature and available for a future mission in order to perform:

- Meteorological measurements

- $\quad$ The measurements of pressure, temperature, wind speed and direction, humidity and dust opacity enable to characterize the basic state meteorology and its daily variation at the landing site.

- Such information can directly be compared to climate models.

- DREAMS is equipped to characterize the Martian boundary layer, also in dusty conditions.

- Hazard monitoring

- DREAMS can provide a comprehensive dataset to help engineers to quantify hazards for equipment and human crew: velocity of windblown dust, electrostatic charging, existence of discharges, and electromagnetic noise potentially affecting communications, intensity of UV radiation.

- Monitor of atmospheric electric phenomena

- A global atmospheric electrical circuit is likely to exist on Mars, between the surface and the ionosphere, with similarities and differences with the Earth’s circuit (Aplin, 2006). Atmospheric ionization should be similar to that of the Earth's stratosphere but impact charging through collisions between dust particles moved by the wind and the surface, or between dust particles themselves, is expected to be the dominant charging mechanism. 
Intense electric fields, possibly capable of producing electrical breakdown, are expected at the time of dust storms and in the vicinity of dust devils.

- Atmospheric electricity is also involved in several processes that have a noticeable impact on the surface and atmosphere. At times of dust storms, electrostatic forces on fine electrically charged dust grains may become larger than aerodynamic forces due to the wind. They are expected to play a significant role in the dynamics (including lifting) of suspended dust particles and their interaction with the surface, thus on the processes that contribute to the erosion and long-term evolution of the surface (Esposito et al., 2016; Harrison et al., 2016; Murphy et al., 2016).

- By energizing the free electrons, the atmospheric electric field control their interaction with both the surface and the atmospheric gases. They have thus a definite role in the chain of physical and chemical processes that govern the chemical state of surface materials and the production of oxidized constituents in the atmosphere with implications on the sustainability of proper conditions for life (Atreya et al., 2006).

A Flight Spare model of DREAMS is mature and available for a future mission.

Some of the DREAMS sensors have been tested in the Sahara desert during the dust storm season to prove their ability to work in a dusty environment and to probe dust events. The field campaign allowed also the advancement of our knowledge of Aeolian processes and their relation with the electric properties of the atmosphere.

The following sections describe the DREAMS instrument design, sensors performance, and calibration procedures. Results of the field tests are described in section 4.

\section{The DREAMS experiment}

The DREAMS experiment is the result of a cooperation of six European Countries (Italy, France, Spain, Netherlands, Finland, United Kingdom) led by Italy. The team organization is described in Figure 1. 


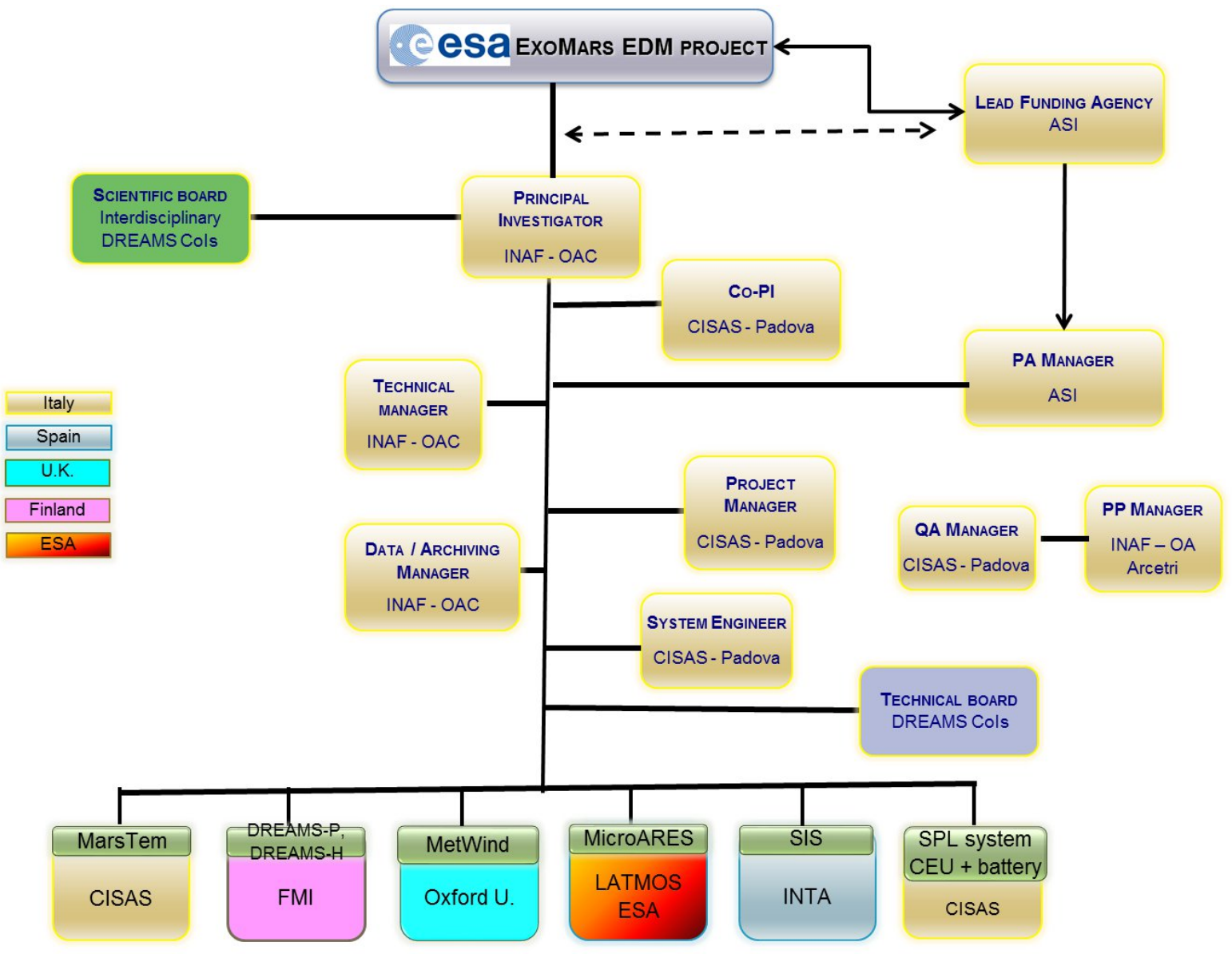

133 Figure 1: DREAMS team organization.

135 DREAMS (Figure 2) is a completely autonomous sensor suite containing the following subsystems:

136 a) the power unit (a space qualified rechargeable battery assembly developed by ABSL), b) the 137 Central Electronic Unit (CEU), comprising all electronic boards for sensor data acquisition and 138 communication with Schiaparelli module, c) the MetMast assembly (about $20.4 \mathrm{~cm}$ tall) which 139 hosts most of the external sensors and d) the MicroARES electrode (27 cm tall). 

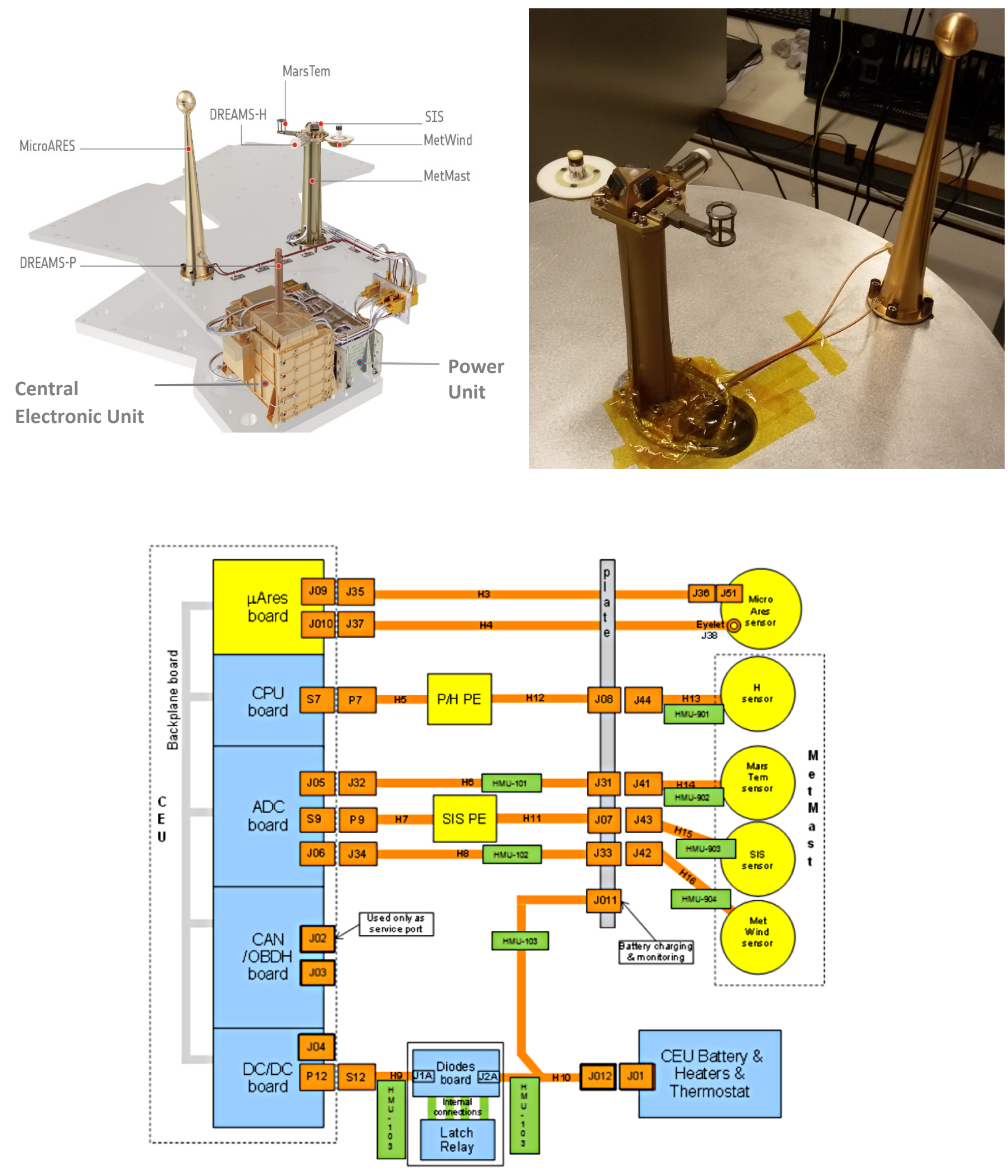

143 Figure 2: Top left: graphic impression of the DREAMS instrument and its location in the 144 Schiaparelli Central Bay. Credit: ESA. Top right: DREAMS spare model: MetMast (on the left) and 145 MicroARES antenna (on the right). Bottom: DREAMS block diagram. 
147 A dedicated harness guarantees the connection of DREAMS hardware located in the internal bay

148 with the external sensing units and the Schiaparelli control unit through a

149 connector bracket which interfaces the internal compartment with the external environment.

150 The following subsections describe each DREAMS element.

2.1 DREAMS-H

DREAMS-H (Figure 3) is a miniature relative humidity measurement device based on technology

DREAMS-H is approx. $15 \mathrm{~g}$ and the power consumption is $15 \mathrm{~mW}$.

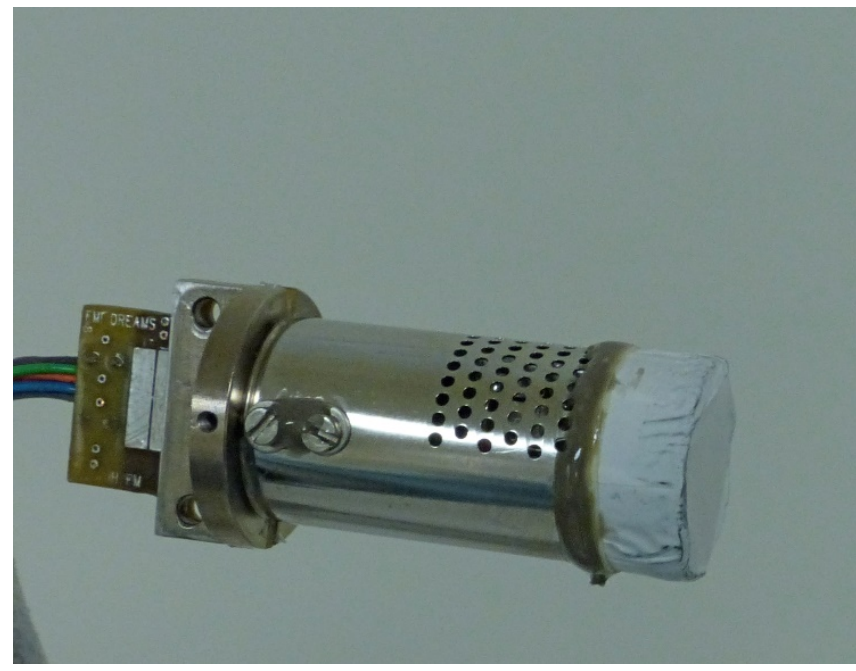

DREAMS - H FM MARCH 2014

Figure 3: DREAMS-H sensor. Credit: FMI/Markku Mäkelä.

As REMS-H, DREAMS-H measures relative humidity with 3 Humicap ${ }^{\circledR}$ polymeric capacitive

sensor heads, while accurate temperature measurements required for interpretation of relative humidity measurements are provided with capacitive Thermocap ${ }^{\circledR}$ sensor heads, both by Vaisala, Inc. The sensor heads are placed on a single PCB together with proximity electronics, and protected with metallic Faraday cage. The cage is perforated to allow gas exchange between the sensor heads and the surrounding environment. To protect the sensor from excessive dust, part of the holes are 
covered with a PTFE filter material. The whole structure is mounted on DREAMS mast at about 17 cm height from the top of Schiaparelli Central Bay. DREAMS-H control and data retrieval is handled by the measurement controller located on the DREAMS-P PCB.

The Humicap ${ }^{\circledR}$ sensor heads contain an active polymer that changes its capacitance as function of relative humidity and temperature with 0 to $100 \%$ RH measurement range. In a given temperature, the response between 0 and $100 \% \mathrm{RH}$ is very close to linear. The polymer reacts to the relative humidity even when the device is not powered, so the relative humidity can be read almost immediately after power-on.

Nominal capacitance of Humicap ${ }^{\circledR}$ is in order of $6 \mathrm{pF}$. The dynamic range of the Humicap ${ }^{\circledR}$ changes with temperature, being approx. $1 \mathrm{pF}$ around 273K and approx. $0.3 \mathrm{pF}$ around 203K. The Humicap ${ }^{\circledR}$ also becomes logarithmically slower with lower temperature, its time constant is about $0.1 \mathrm{~s}$ at $293 \mathrm{~K}$, but for example at $233 \mathrm{~K}$ it is about $30 \mathrm{~s}$ and at $203 \mathrm{~K}$ about $700 \mathrm{~s}$. Some additional time lag is added also by the protective PTFE filter. The time lag caused by the coldness and PTFE filter can be partially mathematically compensated on ground. The compensation algorithm is based on the relative humidity reading and its speed of change. The basis of this method is the fact that the speed of change through the filter depends on the atmospheric temperature and the difference of $\mathrm{RH}$ between the inside and the outside. Therefore, the difference in RH can be calculated by knowing the ambient temperature, and the speed of the change in raw RH values (Harri et al. 2014b). 


\subsection{DREAMS-P}

DREAMS-P is a miniature pressure sensor designed for making accurate measurements in the

Martian atmosphere surface pressure range. The device houses two pressure transducers and an
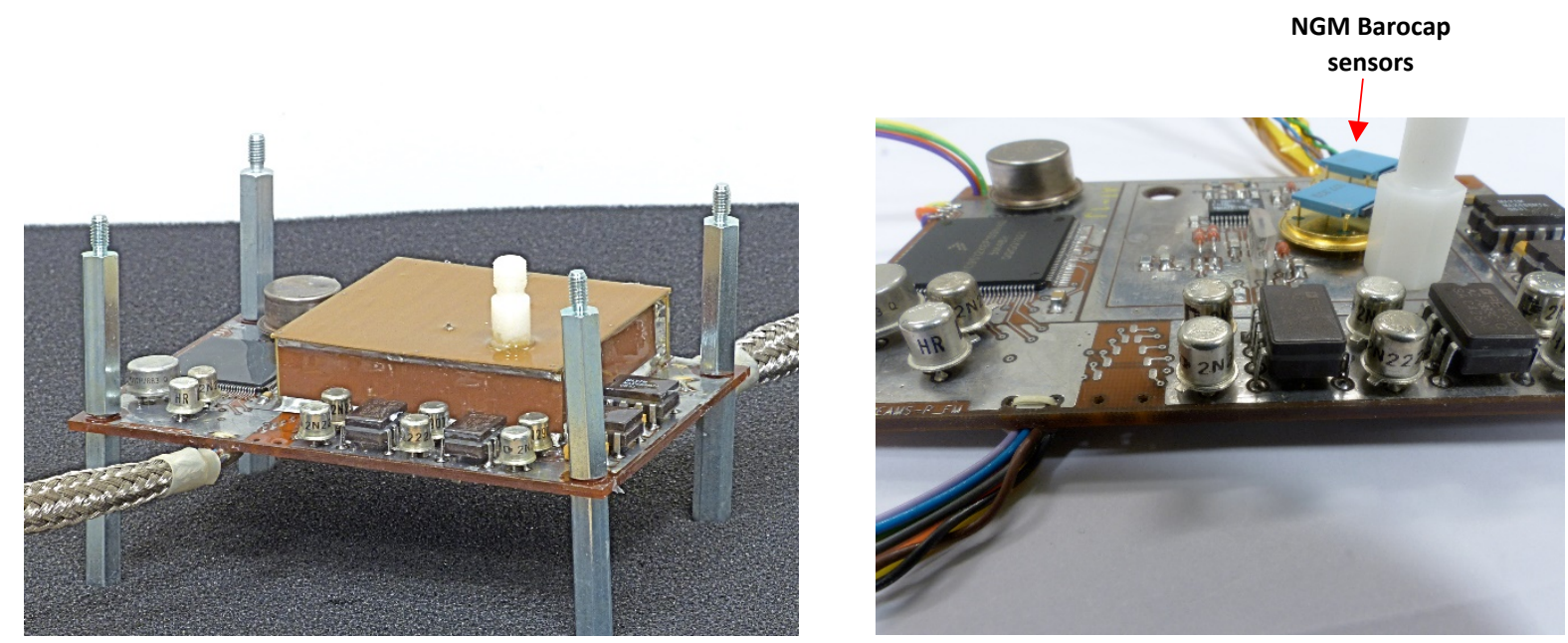

The pressure device is based on capacitive Barocap ${ }^{\circledR}$ sensor heads manufactured by Vaisala, Inc. interference.Atmospheric ambient pressure is led inside the device through a pipe protruding above

Figure 4: DREAMS-P sensor with (left panel) and without (right panel) the cover. Credit: FMI/Markku Mäkelä. The components with blue parts in the right panel are NGM Barocaps. The RSP2Ms are on the other side of the board.

The whole DREAMS-P device is mounted inside the lander warm compartment, with the pressure transducer electronics being housed inside a Faraday shield, a rectangular enclosure made of copper covered Printed Circuit Boards, to protect the electronics from electromagnetic the lander deck. The top part of pipe outlet is designed to prevent excessive amounts of dust from entering the system.

The device consists of two pressure transducers with heritage from the REMS-P instrument flown onboard the Curiosity rover (Harri et al. 2014a). Each transducer has two Barocap ${ }^{\circledR}$ pressure sensor heads, two Thermocap ${ }^{\circledR}$ temperature sensor heads and four constant capacitors for reference. The 
DREAMS-P2 transducer is essentially identical to the electrical design in REMS-P using the

213 Barocap ${ }^{\circledR}$ RSP2M sensor heads, while the DREAMS-P1 transducer utilizes the "new generation”

214 NGM sensor heads by Vaisala, specifically modified for Martian pressure range. This sensor head replaces the now obsolete "LL" sensor head used in Phoenix pressure sensor and REMS. The NGM has no previous flight heritage, but it has been fully qualified for spaceflight during DREAMS testing and qualification campaign.

The pressure dependence of Barocap ${ }^{\circledR}$ capacitance is opposite in RSP2M and NGM. In RSP2M, 
reference crystal clock frequency is utilized to measure the duration of a given amount of sensor or reference channel pulses to derive each measurement channel frequency.

\subsection{MarsTEM}

MarsTEM is a platinum Resistance Temperature Detector (RTD) for the measurement of the atmospheric temperature at the surface of Mars. (Colombatti et al., 2014).

In order to measure the temperature the convective flux is detected by a thin $(\phi=0,0508 \mathrm{~mm})$ and long platinum wire ( $\mathrm{L}=700 \mathrm{~mm}$ ) which lead to a resistance of $37.8 \mathrm{ohm} @ 0{ }^{\circ} \mathrm{C}$ and a time constant of 0.3 seconds at a wind speed of $5 \mathrm{~m} / \mathrm{s}$ and absolute pressure of $6 \mathrm{hPa}$.

The sensor wire is wounded on an insulating structure (Titanium alloy) to minimize the conductive heat flux from the sensor wire and structure. The structure hosts a secondary Platinum RTD, made from the same thin wire of the primary, which provides redundancy (e.g. in case the wounded sensor is damaged by dust). Simultaneous acquisition of both sensors allows for the dynamical correction of primary sensor in data post-processing (Figure 5). In addition, from the retrieved titanium structure time constant, the secondary thermometer will support the correction of errors introduced by solar radiation contribution on the primary sensor. Chiodini et al. (2014) showed that the radiative contribution of the thermal flux on the sensor is lower than the $30 \%$ at noon conditions (the worst from this point of view) with a wind of $1 \mathrm{~m} / \mathrm{s}$ and that this contribution decreases to $20 \%$ with a $5 \mathrm{~m} / \mathrm{s}$ wind.

The radiative flux contribution produces an increase of about $1.5^{\circ} \mathrm{C}$ in the temperature of the sensing element. Anyway, most of this contribution is due to the radiative flux arriving on the sensor from the structure itself and not directly from the sun. Similar increase in temperature has also been observed in the test field performed in the Moroccan desert (Colombatti et al., 2015). Primary and secondary sensors are sensed with a 3-wires configuration circuit allowing conditioning and acquisition of voltage through the DREAMS electronics.

To minimize the self-heating and to achieve anyway high electrical sensitivity, the sensors are powered with a pulsed current profile. In such a way the rms current value is very low, which 
means negligible joule effect, while instantaneous current value is high to maximize the sensors electrical sensitivity. MarsTEM design and realization, including its conditioning circuitry, were validated through the Missus Experiment flown on BEXUS15 ESA/SSC/DLR stratospheric balloon, which hosted the sensor prototype and the conditioning circuitry.

The terrestrial absolute pressure at about $30 \mathrm{~km}$ of altitude is an analogue of Martian pressure at the surface. Missus allowed the validation of the mathematical model (even of sun irradiance effects) and the tuning of the electrical current profile in order to maximize sensitivity/self-heating ratio and the overall SNR. To infer MarsTEM performance from MISSUS experiment results, the comparison between Earth and Mars potential temperature was performed (Chiodini et al., 2014). MarsTEM performances were obtained during calibration within the range $-100{ }^{\circ} \mathrm{C}+20^{\circ} \mathrm{C}$.

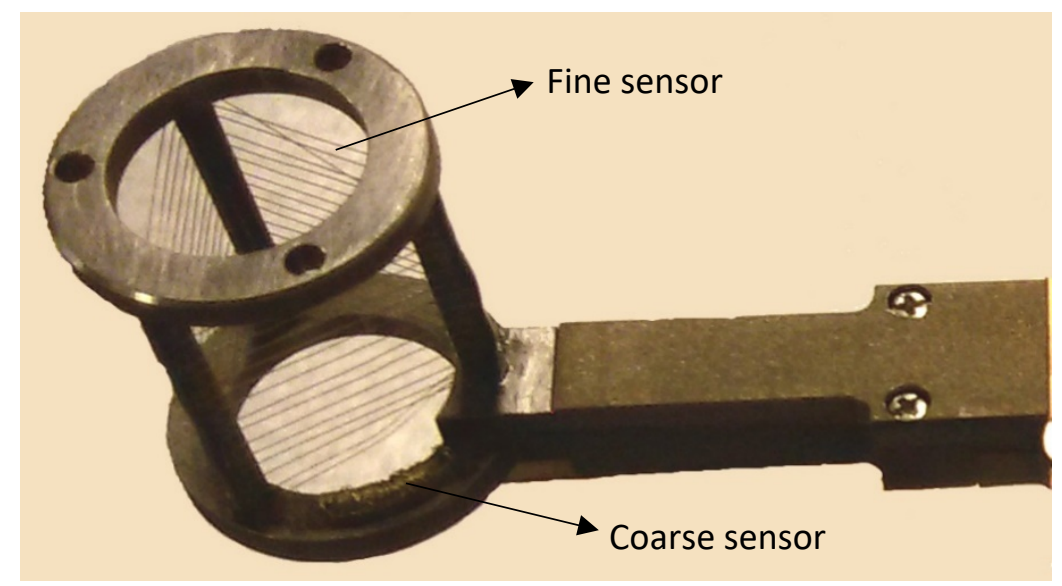

Figure 5: MarsTEM sensor with the fine and coarse subsystems indicated.

\subsection{MetWind}

MetWind is a thermal anemometer that measures the components of wind speed and direction in the plane perpendicular to the sensor axis. The sensor head consists of three thin-film platinum heat transfer gauges, equally spaced around the circumference of a vertical cylinder (Figure 6). Each film is resistively heated using a constant current raising its temperature above the ambient air. The film's electrical resistance is measured, allowing calculation of the temperature and thus the heat transfer coefficient at each hot film. The differences in heat transfer coefficients between the three 
films is used to calculate a two-dimensional wind vector perpendicular to the axis of the wind

sensor. In its normal orientation the axis of the central cylinder is vertical, so that the sensor

wind speeds above this range can be measured as well.

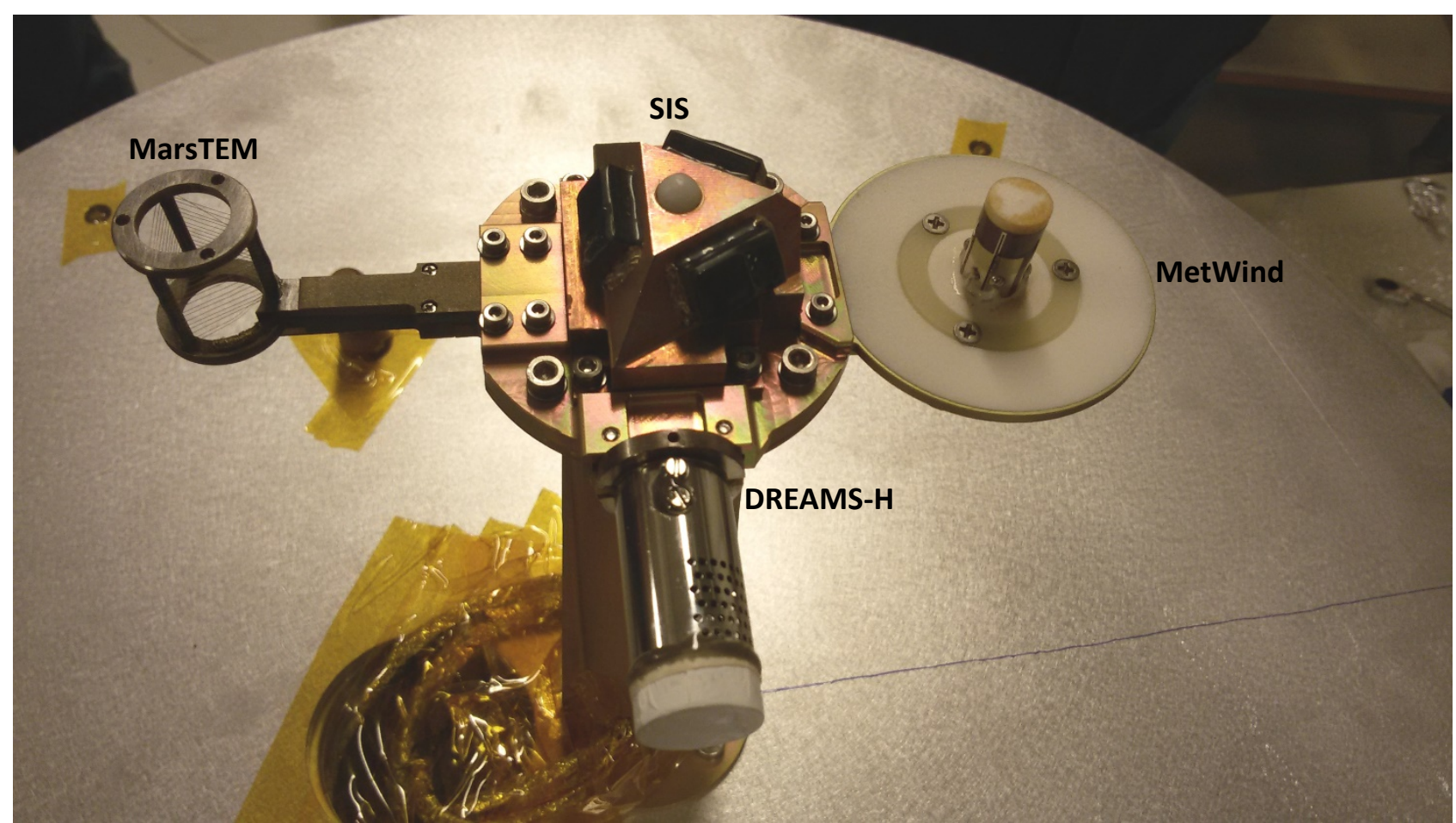

Figure 6: MetMast top view.

Thermal wind sensors have a long heritage on Mars. These include the Viking Lander wind sensors;

the Mars Pathfinder wind sensor, Mars Polar Lander wind sensor, and the Mars Science Laboratory wind sensor. Principle advantages of thermal anemometry are the low mass and simplicity of thermal wind sensors. Balanced against this advantage is the fact that thermal wind sensors are difficult to calibrate, because they are sensitive not only to wind but also to other thermal loads, such as those caused by changes in air temperature and solar illumination. MetWind reuses the Beagle 2 Wind Sensor design (Towner et al., 2004; Wilson et al., 2003), with the addition of a housekeeping sensor: a platinum resistance thermometer that has been placed in the center of the sensor. The data from this thermometer supports the calculation of the conductive 
heat losses, which would otherwise be one of the largest sources of uncertainty in interpreting the wind speed data.

The MetWind thermal anemometry technology is similar to that used on Mars Pathfinder (Seiff et al., 1997). Wind speed data acquired by the Mars Pathfinder sensor has not been published because of large uncertainty in the overheat $\left(T_{\text {wire }}-T_{\text {air }}\right)$, which in turn was caused by large fluctuations in the air temperature (up to $\pm 10^{\circ} \mathrm{C}$ during the day). This air temperature fluctuation is large in comparison to the overheat of the sensor wire, which was only $1-10^{\circ} \mathrm{C}$ for the Pathfinder sensor. The overheat of the Beagle 2 wind sensor's hot films would have been typically $50-70^{\circ} \mathrm{C}$, and for MetWind this was increased to $70-85^{\circ} \mathrm{C}$ by adjusting resistances; this greater overheat reduces the measurement error in wind speed.

The MetWind electronics are designed to allow two sensing modes. The first is a low-current mode ('TEMP' mode), in which only $1 \mathrm{~mW}$ of heating power is dissipated in the films. In this mode the self-heating is only $\sim 2^{\circ} \mathrm{C}$, and is relatively insensitive to wind speed. In this mode, therefore, the wind sensors can be used to provide a backup measurement of air temperature; it also provides a way of check whether the film resistances have changed with time, or example due to sand abrasion.

On power-up, the electronics are typically started in the low power (TEMP) mode. After 30 seconds, MetWind is switched to high power mode (WIND) mode, in which $\sim 40 \mathrm{~mW}$ are dissipated in each film. The electronics then remain in WIND mode until they are next powered off and back on again.

The response time of the sensor to step changes in wind speed is $\sim 2-5$ seconds, dependent on wind speed and direction. This is sufficient to allow characterization of large-eddy turbulence and detection of convective vortices.

MetWind is extremely light: the sensor head itself weighs only 0.7 grams. Once its mounting screws and $400 \mathrm{~mm}$ harness are taken into account the total mass is 11 grams, excluding connectors 
and electronics (which are included in the CEU). Total power consumption while sensing wind is around $250 \mathrm{~mW}$, of which $120 \mathrm{~mW}$ are dissipated in the sensor head.

\subsection{MicroARES}

Micro-ARES is a single probe electric field instrument consisting of a spherical electrode installed on a stiff metallic support (Figure 2) and a single electronics board housed in the common electronics box (DREAMS CEU) placed in the warm compartment.

The predecessor of MicroARES called ARES (proposed in a double probe version, see for instance Berthelier et al., 2000) has been validated on two balloon flights and was developed as part of the HUMBOLDT platform payload (and successfully passed the Preliminary Design Review) in a previous configuration of the ExoMars mission.

MicroARES measures the amplitude of the vertical component of the electric field in the atmosphere, with the lander potential as reference. A high impedance $\left(10^{14} \Omega\right)$ preamplifier is mounted in a voltage follower configuration yielding a precise measurement of the surrounding atmospheric potential; the Mars near surface atmosphere is a medium whose typical resistivity is $>10$ smaller than the MicroARES instrument impedance (Farrell et al., 2015).

In the Analog portion of the electronics board, the signal is separated in two components: (1) the large amplitude ( $\mathrm{mV}$ to $\mathrm{V}$ ) and low frequency $(<10 \mathrm{~Hz})$ signal of the DC channel and (2) the small amplitude $\left(10^{-3} \mathrm{mV}\right)$ and high frequency $(100 \mathrm{~Hz}$ to $\mathrm{kHz})$ signal of the AC channel. The difference between the two resides in the capacitive coupling of the AC channel with the antenna, which effectively suppresses the main component of the signal received from the sphere. The high sensitivity of the AC channel can be used to detect the impacts of charged dust particles to let one infer their horizontal flux and charge distribution.

A high voltage mode is automatically activated by the Micro-ARES central computer (a Texas Instrument ${ }^{\circledR}$ Digital Signal Processor, hereafter referred as to DSP) when the modulus of the acquired potential exceeds $90 \mathrm{~V}$ for a certain amount of time (typically a fraction of second). This high voltage 
mode disrupts the equilibrium of potentials between the antenna and the atmosphere by bifurcating the input signal into a resistive bridge dividing the signal by a factor of $>50$ and forcing it to cope with the voltage range admitted by the 16 bits ADC.

When operated in the relaxation probe mode, the instrument measures of the atmospheric conductivity separately for positive and negative ions. Periodically, $+1 \mathrm{~V}$ and $-1 \mathrm{~V}$ pulses are sequentially injected by capacitors into the antenna, creating a small and temporary departure from equilibrium between the potential of the antenna and that of the atmosphere surrounding it. Positive and negative ions flowing around the electrode are then attracted by the antenna surface depending on the pulse polarity to fill in the gap of potentials with a characteristic e-folding relaxation time. This provides data for estimating the positive and negative conductivities.

MicroARES computing capabilities are ensured by a Digital Signal Processor (DSP) from Analog Devices ${ }^{\circledR}$ (model ADSP-2189). This DSP has a 13 ns instruction cycle time and can handle 75 MIPS. It is particularly notable for its large on-chip memory (1.5 MBits) and its low power features (2.5v). As the ADSP-2189 cannot be procured in a space-qualified version, it received the needed screening and qualification (Construction analysis, Highly Accelerated Stress Tests, Life test, Single Event Effects and Total Ionisation Dose testings) to verify that proper operations would be carried out once at Mars. The main DSP tasks are setups and commands of the analog part, real time signal processing (data decimation, filtering, selection and conditioning), and ensures communication with the CEU.

\subsection{SIS}

SIS (Solar Irradiance Sensor) is a radiometer designed to measure solar irradiance on the Martian surface. Its main goals are: (a) to provide an estimation of the atmospheric optical depth (OD) and its variations within a Sol; (b) to allow for the detection of clouds through the analysis of the shape of the ratio between the signals measured in two different spectral bands (UV and NIR) during twilight; and (c) to provide a direct measurement of the global irradiance on the surface. 
It consists of two units, an optical head $(\mathrm{OH})$ and a processing electronics (PE) box. OH contains the detecting elements and front-end electronics, whereas PE acquires and digitizes the signals provided by the $\mathrm{OH}$, controls the sensor and communicates with the $\mathrm{CEU}$. OH is located on top of the DREAMS MetMast (Figure 2, Figure 6). This placement severely constrained the OH mass to less than 26g. $\mathrm{OH}$ contains 7 different silicon photodetectors distributed on 4 faces: one bare silicon detector with a spectral response of 200-1100 nm pointing to the instrument's zenith, and three pairs of "lateral" detectors covered with filters in the UV and NIR bands (UV: 315-400 nm; NIR: 700$1100 \mathrm{~nm}$ ), pointing to an elevation of 30 degrees and azimuths of 60, 180 and 300 degrees within the $\mathrm{OH}$ reference frame. The detectors are commercial-of-the-shelf photodiodes that are covered with custom interference filters to provide the desired spectral response. $\mathrm{OH}$ contains an eighth detector inside its mechanical assembly, covered by a lid to inhibit light reception. The dark current of this detector is measured to estimate the displacement damage suffered during the trip to Mars (Jiménez et al., 2012). Each lateral detector is covered by an interference filter and a mechanical mask that provides the desired Field-of-View (FoV). This FoV is around \pm 40 degrees when measured to a $10 \%$ of the maximum responsivity. The zenith detector is covered with a diffusing dome that provides a quasi-planar hemispherical FoV. The signals of the SIS detectors, together with some housekeeping signals, are conditioned and multiplexed within the OH. Small footprint, precision, low-noise, low-power and Rail-to-Rail COTS (Commercial-Of-The-Shelf) operational amplifiers were selected, qualified and screened for the Front-End SIS electronics. Tests of these parts included radiation (TID, up to 30 krad) (Álvarez et al., 2014), vibration, shock, thermal cycling and operation in extreme temperature (down to $-135^{\circ} \mathrm{C}$ ). The photodetectors and the optical elements were also tested using similar procedures. 

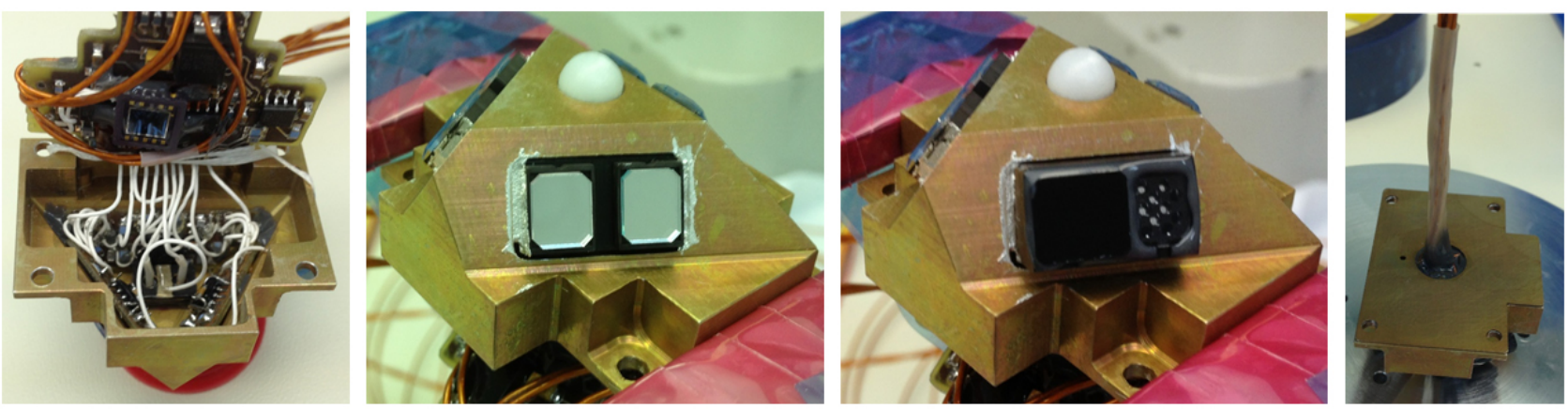

401

402

403

404

405

406

407

408

409

410
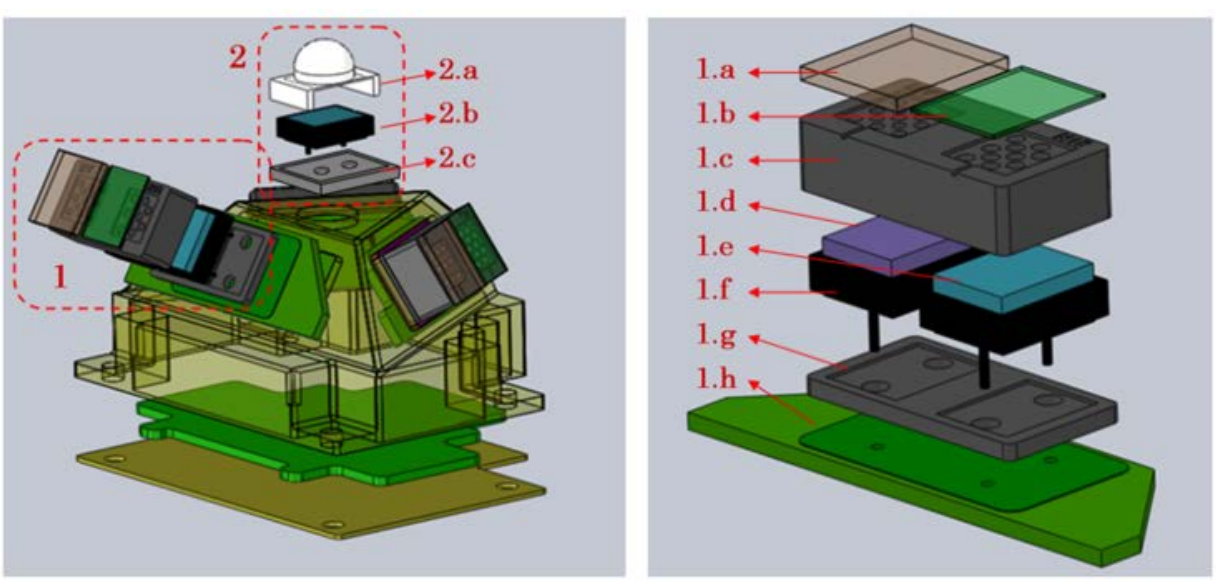

Figure 7: Top: Views of SIS-OH during the integration process. Bottom: Detail of the Optical Head elements. 1.a, b: external UG-11 (on the UV detector) and Teflon sheet (NIR one) covers. 1.c: FoV-shaping element. 1.d,e: interference filters. 1.f: silicon photodiodes. 1.g: radiation shield (added to ensure a minimum thickness of aluminum for radiation protection). 1.h: printed circuit board with the current-to-voltage amplifiers. 2.a: diffusing dome covering the unfiltered detector. 2.b: silicon photodetector (without any filter). 2.c: radiation shield.

The PE is a small processing unit that performs the analog-to-digital conversion of the signals received from the $\mathrm{OH}$, plus some others internally generated at $\mathrm{PE}$, stores the resulting information, and communicates with DREAMS CEU in order to control the operation of SIS and to send the resulting data packets. The unit is based on space grade parts, being its core an anti-fuse FPGA. PE offers two possible operation modes, namely Manual and Automatic. In the Manual mode, the unit is always the slave in a Master/Slave communication in which the CEU must command a new acquisition and data returning each time it wants to get some data from SIS. In the Automatic mode, the CEU commands SIS to perform automatic acquisitions of all its signals without further intervention of CEU and according to a desired sampling period form. Then SIS operates 
autonomously storing data on its internal memory (up to $128 \mathrm{kB}$ ), until it is requested to stop and

419 dump the data to CEU, or until the memory gets full.

420 PE also incorporates a MEMS (Micro Electro-Mechanical System) accelerometer to determine the

421 tilt of the lander on the surface of Mars. This is also a COTS part that was qualified and screened

422 for use in DREAMS. Intensive tests include radiation, vibration, shock, vacuum, and thermal

423 cycling (Álvarez et al., 2015).

424

425

426

427

\subsection{CEU}

The CEU (Central Electronic Unit) is a modular unit (Figure 8) designed to provide all primary functionalities needed for operation, data acquisition and communication as timeline-based environmental operative sequences, analogue to digital conversion for the analogue environment sensors, power transformation and supply for all sensors/conditioners. The unit also performs data compression to meet the requirements on data volume budget for the ExoMars mission and realizes data packetization for telemetry transfer to the EDM on board computer using non-volatile mass memory for data storage.
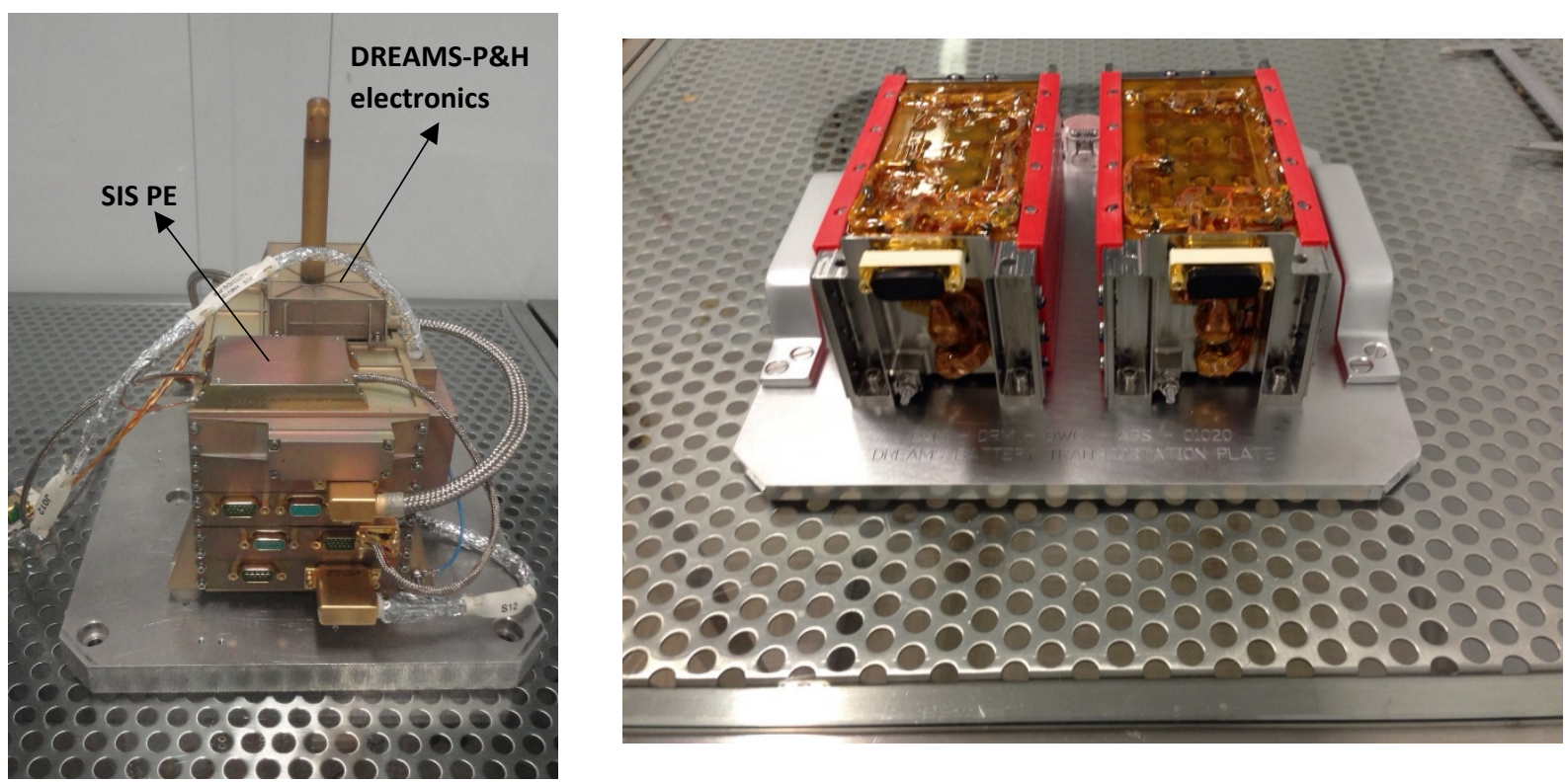

Figure 8: Left: CEU FM; right: two models of the battery units. 
435 The CEU is able to execute self-test and to provide information of status and sensors health during in 436 flight checkouts.

437 The modular design is based on a plug-in configuration, splitting functionalities into different boards 438 connected through a common backplane; the boards installed on the ExoMars 2016 flight unit are:

439 - OBDH Board implements the control layer of the CEU: stores and applies the Data Acquisition 440 Timelines, interprets and forwards the telecommands coming from EDM to the peripheral boards 441 (including data on power source use), collects the housekeeping data, acquire sensors data, stores 442 HK and sensor data in a devoted 4 Gbit nonvolatile memory and uploads the data to the EDM 443 for transmission to relay orbiter and then back to Earth;

- DC/DC Board performs the power distribution to lines of CEU boards and of each sensor 445 according to the profile of each mission phase;

446 - CPU Board compresses high volume data with dedicated algorithms depending on specific 447 structure of data packets. This board also incorporates all the physical layers of the interfaces $448 \quad$ towards the MicroARES board and DREAMS P/H sensor;

449 - MicroARES Board is integrated in a dedicated slot of CEU, and performs the control of power $450 \quad$ and data handling of the MicroARES sensor;

451 - ADC Board performs conditioning and acquisition of signals of analogue sensors depending on 452 operative timelines. The data collected are transferred to the OBDH Board for storage and 453 transmission.

- The CEU assembly hosts a relay module used to switch the power from the main bus to the 455 DREAMS own Battery. In addition a devoted timer, which convert UTC into Mars Local time, is 456 operative all along the scientific mission. It synchronizes the data communication from to lander 457 electronics, and it allows the Mission Timeline execution.

458 Overall dimensions are $180 \mathrm{~mm}$ x $151 \mathrm{~mm}$ x $136 \mathrm{~mm}$ with a total mass for the complete flight model 459 of $2219 \mathrm{~g}$. 
DREAMS power source for autonomous operation on Mars is a rechargeable battery (Figure 8), based on Li -Ion rechargeable cells arranged in 8s3p lay-out, containing 3 strings in parallel, each consisting of 8 cells in series. Nominal voltage for fully charged battery is $33.6 \mathrm{~V}$ and an overall battery capacity of 6.9 Ah.

Charging and discharging sequences before launch are realized through a 7 pin micro connector installed on Warm Compartment connector bracket, using dedicated ground support equipment compatible with ISO 7 environment specifications. Last battery charging for DREAMS flight unit was performed on January $20^{\text {th }} 2016$ in Baikonur before closing Schiaparelli for final preparation for 470 launch.

471 Overall battery envelop is $200 \mathrm{~mm}$ x $100 \mathrm{~mm}$ x $95 \mathrm{~mm}$ with a total mass of flight unit of $1691 \mathrm{~g}$. 472 Mechanical structure of battery includes heaters and thermostats (nominal and redundant) to keep the battery temperature within the required temperature range $\left(-17^{\circ} \mathrm{C},+50^{\circ} \mathrm{C}\right)$, while controlling relays are mounted on a side of CEU unit and commanded through the CEU DC/DC board.

475 Lifetime prediction of the battery for nominal operation on Mars is around two sols taking into 476 account charge depletion during launch preparation and cruise to Mars and the power needed for temperature control.

The DREAMS power consumption has been measured during environmental testing for all possible operational states foreseen during cruise and on Mars surface; at the nominal battery voltage of 28V an average power consumption of $5.60 \mathrm{~W}$ has been measured in Cruise and Surface states, that rises to $7.84 \mathrm{~W}$ during acquisition, a consumption of $6.72 \mathrm{~W}$ in Upload state and of $0.28 \mathrm{~W}$ in idle state. The DREAMS overall mass, as measured on the Flight Model, is $4362 \mathrm{~g}$. 


\subsection{Calibrations}

DREAMS sensors have been calibrated individually by the various lead team members. The overall calibration has been then verified at the DREAMS project level.

\subsubsection{DREAMS-H}

491 Calibration of DREAMS-H has been done in similar way as calibration of REMS-H (Harri et. al. 492 2014b). Three flight grade models, Flight (FM), Spare (FS), and Ground reference (REF), were 493 manufactured at the same time, using parts from the same lots, and calibrated simultaneously. 494 Temperature calibration of Thermocap ${ }^{\circledR}$ sensor heads has been performed at several stable 495 temperatures between $203 \mathrm{~K}$ and $333 \mathrm{~K}$. The resulting accuracy of the temperature readings by 496 Thermocap ${ }^{\circledR}$ is better than $+/-0.1 \mathrm{~K}$ compared to the used reference Pt100 sensor, which in turn has 497 calibration traceable to national standards.

498 For room temperature measurements, a simple humidity calibration function without temperature 499 compensation was determined from the measurements made in several humidity points in 295K 500 temperature. This simple calibration function is only to be used for interpreting measurements made 501 close to the room temperature (above 283K), with approx. +/-4\% RH accuracy.

502 For low temperature $(<273 \mathrm{~K})$ measurements, DREAMS-H dry $(0 \% \mathrm{RH})$ calibration curve was 503 established from measurements performed in vacuum in the temperature range of 203...293K, and $\mathrm{H}$ between dry and saturation points more accurately than with a simple linear fit, intermediate humidity points of approx. 30\%, 60\%, and 90\%RH w.r.t. ice were also measured in the calibration facility of the Finnish Metrology Center (VTT) in several stable temperature points between 203K and 263K. To determine the response of DREAMS-H in changing humidity and temperature conditions, also measurements in varying conditions (so called "Mars sol simulation”) were 
performed. The main calibration was done in ambient pressure, air, except for the dry curve which was done in vacuum. The $\mathrm{CO}_{2}$ effect on Humicap capacitance was measured separately. Indeed, $\mathrm{CO}_{2}$ affects the capacitance of the Humicaps, the effect being larger in high pressure and cold temperature. In Martian pressure range the effect is negligible for temperatures above 263K, but in colder cases it has to be compensated. For this, dry point curve of DREAMS-H ground reference model (REF) was measured also in $8 \mathrm{hPa} \mathrm{CO} 2$ environment in the temperature range of 203...293K, and compared to the curve measured in vacuum. Based on these measurements, the temperature dependent offset caused by $\mathrm{CO}_{2}$ for the dry curve was calculated. The offset was of the same order for all 3 Humicaps on DREAMS-H REF, and also for the Humicaps on REMS-H REF device, for which dry point comparison in vacuum and 8hPa $\mathrm{CO}_{2}$ was also made. The DREAMS-H Humicap capacitances measured on Mars shall be adjusted by this offset before calculating calibrated humidity values. Also wet point verification measurements with DREAMS-H and REMS-H reference models in low pressure $\mathrm{CO}_{2}$ environment are under way.

The details of the calibration procedure will be published in a separate paper.

With the calibration based on the measurements made at FMI and Finnish Metrology Center, and using the mathematical compensation for the Humicap ${ }^{\circledR}$ chip lag in cold temperatures and the PTFE filter effect, the accuracy of humidity measurements in changing temperature and humidity conditions is better than $+/-10 \% \mathrm{RH}$ in the temperature range of $203 . . .273 \mathrm{~K}$, and in order of $+/-$ $20 \% \mathrm{RH}$ in the temperature range of $190 \ldots 203 \mathrm{~K}$. In dry conditions $(\mathrm{RH}<10 \%)$, better accuracy of +/-4\%RH over the whole operational temperature range is achieved.

\subsubsection{DREAMS-P}

The calibration of DREAMS-P was performed in August 2014 at FMI, in air environment. The Barocap measurement principle is based on the medium gas pushing the capacitance plates closer or further away from each other. This plates movement is not affected by $\mathrm{CO}_{2}$. There is no polymer in this case, that would react to different gases as with Humicap sensors. In the sensor level calibration 
the output of the pressure sensor was measured in several pressure and temperature points under stable and under changing temperature. The stable temperature measurements were performed in the range of $0-1400 \mathrm{~Pa}$ (vacuum to Martian pressure range) with $100 \mathrm{~Pa}$ intervals and in $-45^{\circ} \mathrm{C}$ to $+55^{\circ} \mathrm{C}$ temperature (operational temperature range inside DREAMS CEU box) with max $15^{\circ} \mathrm{C}$ intervals. In the measurements under changing temperature, pressure was kept at $800 \mathrm{~Pa}$ while temperature was swept over the operational range down and up, once with as fast rate as possible, and once slower, simulating an actual sol in the mission based on thermal modeling of the CEU. A Vaisala PTB201 pressure transmitter modified for the Martian pressure range and MKS Baratron 10 Torr pressure transmitter were used as pressure references. The accuracy of the reference sensors is $0.2 \mathrm{~Pa}$ and their calibration is traceable to national standards. The reference temperature was measured with Pt100 sensors with calibration also traceable to national standards.

Based on results of environmental tests and experience from previous planetary missions, it was known that the pressure dependence of Barocap ${ }^{\circledR}$ sensor heads is extremely stable, but small changes in temperature dependence and offset may occur, especially after integrating the sensor inside the CEU. To determine these changes, calibration check was performed in vacuum at DREAMS level, after integrating DREAMS-P in CEU. Several calibration checks were also performed during the interplanetary cruise using vacuum as reference. Readings measured in the last cruise check-out were used to compensate the offset drift during storage and cruise (as reported in Sect. 3.2).

Based on the calibration checks performed at sensor and DREAMS level, it can be concluded that in general, in both transducers, Barocaps on channel 2 have better performance than Barocaps on channel 1, so they should primarily be used for scientific purposes. The total accuracy of Barocap \#2 is 2.7 Pa for DREAMS-P1, and 3.2 Pa for DREAMS-P2. The repeatability, meaning maximum artificial variation on diurnal time scale (peak to peak), is $1.4 \mathrm{~Pa}$ for $\mathrm{P} 1$ and $2.5 \mathrm{~Pa}$ for $\mathrm{P} 2$, and the resolution $0.1 \mathrm{~Pa}$ for $\mathrm{P} 1$, and $0.5 \mathrm{~Pa}$ for $\mathrm{P} 2$. These performance parameters are valid after the sufficient warm-up time has passed from the powering of each oscillator (8 min for P1, 2 s for P2). 
Table 1 summarizes the performances of DREAMS-P.

\begin{tabular}{|l|l|l|l|l|}
\hline Oscillator & \multicolumn{2}{l}{$\begin{array}{l}\text { 1 } \\
\text { DREAMS-P1 } \\
\text { (NGM) }\end{array}$} & \multicolumn{2}{l|}{$\begin{array}{l}\text { 2 } \\
\text { DREAMS-P2 } \\
\text { (RSP2M) }\end{array}$} \\
\hline Channel & 1 & 2 & 1 & 2 \\
\hline $\begin{array}{l}\text { Repeatability = Maximum artificial variation } \\
\text { on diurnal time scale (peak-to-peak) }\end{array}$ & $4.3 \mathrm{~Pa}$ & $1.4 \mathrm{~Pa}$ & $6.5 \mathrm{~Pa}$ & $2.5 \mathrm{~Pa}$ \\
\hline Absolute accuracy & $5.0 \mathrm{~Pa}$ & $2.7 \mathrm{~Pa}$ & $7.2 \mathrm{~Pa}$ & $3.2 \mathrm{~Pa}$ \\
\hline Resolution (peak-to-peak) & $0.1 \mathrm{~Pa}$ & $0.1 \mathrm{~Pa}$ & $0.4 \mathrm{~Pa}$ & $0.5 \mathrm{~Pa}$ \\
\hline Warm-up time & $6 \mathrm{~min}$ & $8 \mathrm{~min}$ & $2 \mathrm{~s}$ & $2 \mathrm{~s}$ \\
\hline
\end{tabular}

565

Table 1: DREAMS-P performances.

\subsubsection{MarsTEM}

At sensor level the calibration of MarsTEM has been done in a thermal bath, filled with liquid ethanol, with a stability of $0.03 \mathrm{~K}$ and by performing a comparison between the MarsTEM and a calibrated platinum RTD whose accuracy is better than $0.05 \mathrm{~K}$ in the foreseen operative range. The calibration is performed in the range $-120^{\circ} \mathrm{C} \ldots+40^{\circ} \mathrm{C}$. The accuracy estimation includes other effects such as the Seebeck on in the junctions and soldering. Connecting wires between MarsTEM and CEU have been selected to have low Seebeck effect with platinum sensitive element.

A second step calibration was also performed at DREAMS level (both in vacuum and at $6 \mathrm{hPa}$ between $-120^{\circ} \mathrm{C}$ and $+55^{\circ} \mathrm{C}$ ) in order to take into account the effects due to the electrical resistance of the cables and their variation versus temperature.

Final accuracy and resolution for MarsTEM are $0.1 \mathrm{~K}$ and $0.02 \mathrm{~K}$, respectively.

Self heating effect for the MarsTEM was considered at design level (Vidali et al., 2012). A mathematical model of MarsTEM representing the sensitive element (platinum wire) was developed and tested with several different ambient profiles in order to choose the wire diameter, the insulation thickness, the housing material. An electrical current profile was studied in order to reduce as much as possible the self-heating with a duty cycle of $23 \%(15 \mathrm{~ms}$ ON - 50ms OFF) with a $1 \mathrm{~mA}$ current. The maximum increase in temperature observed was around $0.001 \mathrm{~K}$ (after $80 \mathrm{~s}$ ) in a typical Martian-like environment ( $\mathrm{CO}_{2}$ atmosphere at 6 mbar). Sampling is set at $0.2 \mathrm{~Hz}$. 
A study was also performed in order to investigate and quantify the flow distortion due to the presence of Schiaparelli lander (MarsTEM is accommodated at about $200 \mathrm{~mm}$ from the deck) and MetMast shape and the temperature perturbation due to the buoyant thermal plume from the lander itself. The complete results of the thermal and Computational Fluid Dynamics (CFD) of the lander can be found in Chiodini et al. (2014).

Results of the simulation are displayed in Figure 9, where the difference in temperature measured at the MarsTEM locations versus the incoming fluid temperature, for different wind velocities and a deck temperature of $231.5 \mathrm{~K}$ are shown. Thermal plume in low wind conditions led to a circulation around the lander, which modifies wind direction and speed around the lander.

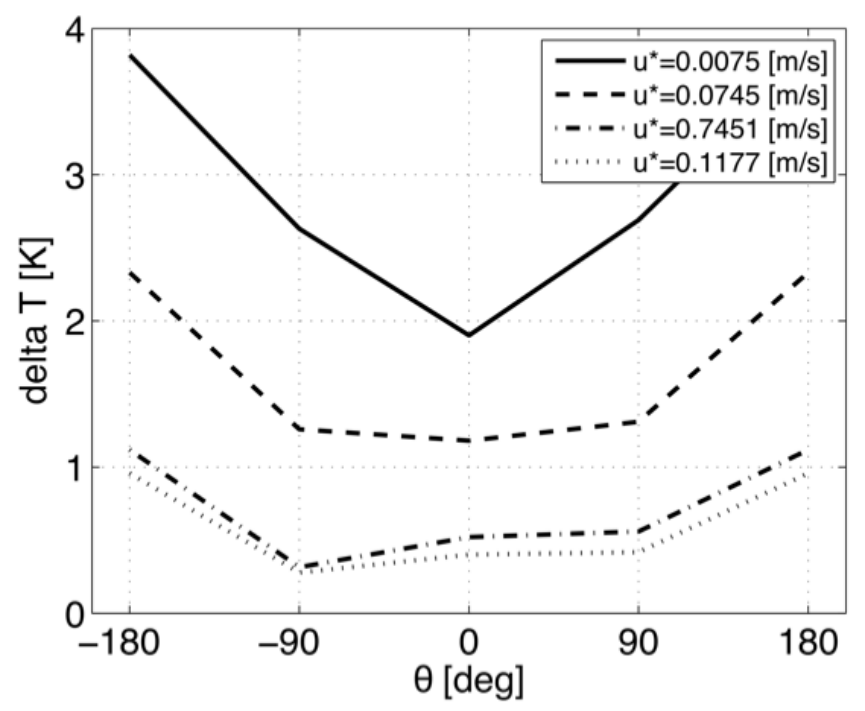

Figure 9: Difference between flow temperature at MarsTEM location and free stream. $\mathrm{u}^{*}$ is the friction speed corresponding respectively to $0.1,1,10$ and $15 \mathrm{~m} / \mathrm{s}$ incoming wind velocities.

The experimental verification of the flow distortion has been performed at the Martian Wind Tunnel in Aarhus (Holstein-Rathlou et al., 2014). These results will be presented in a separate paper.

\subsubsection{MetWind}

The MetWind calibration included the following steps:

1. Resistance vs Temperature calibration

2. Wind tunnel calibration: 
o Measurement of sensor output as function of wind angle (Oxford Mars wind tunnel)

o Measurement of sensor output in varying wind speeds (Oxford Mars wind tunnel)

o Measurement of the shadowing effects by the MetMast (Aarhus Mars wind tunnel)

MetWind sensor was subjected to a thermal soak in the temperature range of $-77^{\circ} \mathrm{C}$ to $+127^{\circ} \mathrm{C}$.

A linear fit of the form $R(T)=R_{r e f} *\left(1+\alpha\left(T-T_{r e f}\right)\right)$ was found to describe the temperature dependence of resistance of each of the three platinum films. $\mathrm{R}$ is the film resistance, $\mathrm{T}$ is temperature, $\mathrm{R}_{\mathrm{ref}}$ and $\mathrm{T}_{\mathrm{ref}}$ are respectively the resistance and temperature of the PTR sensor (Rosemount MF0118 PRT500) used as reference, $\alpha$ is a calibration parameter.

\section{Wind Tunnel Calibration}

The first two tests were carried out in Oxford's Low Density Wind Tunnel facility described in Wilson et al. (2008). The MetWind sensor was placed inside the wind tunnel on the axis of a stepper motor, allowing it to be rotated around its axis of symmetry to simulate different wind directions. All tests were conducted in air at room temperature and at 6 mbar pressure. Scaling to Mars conditions is achieved by using scaling laws with non-dimensional parameters: Reynolds number $R e$ for wind speed and Nusselt number $\mathrm{Nu}$ for heat transfer. Discussion of the validation of the calibration approach, demonstrating the effectiveness of the scaling laws for different gases and varying pressures, is discussed by Wilson (2003b). Measurements were performed both in 'TEMP' and 'WIND' modes with the sensor placed at different angles with respect to the wind direction and at different wind speeds (including no wind). Measurements were obtained by averaging measurements acquired while rotating the sensor from $0^{\circ}$ to $360^{\circ}$ and vice versa in order to correct for any hysteresis associated with lag in the response of the sensor to changing wind direction. Sample results for the MetWind flight model are shown in Figure 10. 


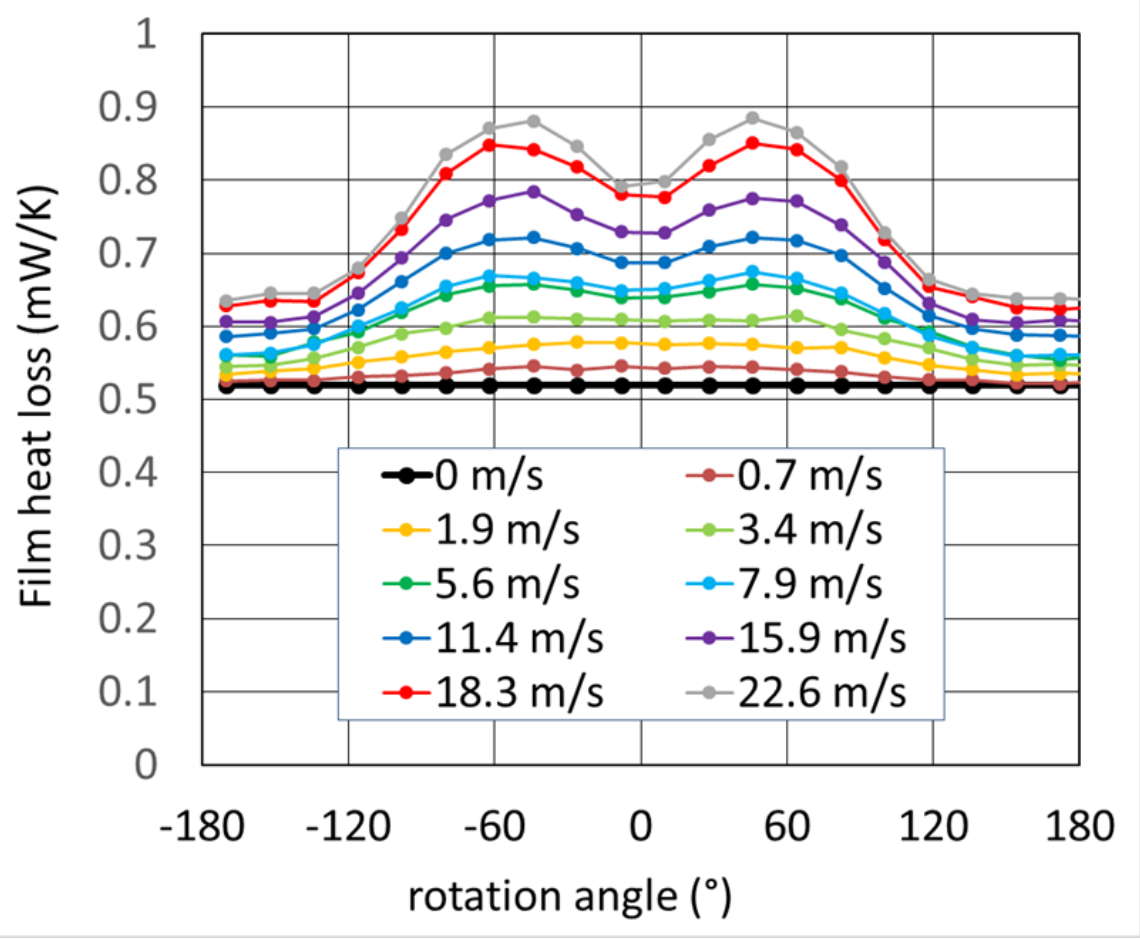

631 Figure 10: Calibration data for one of the MetWind Flight Model sensor films. The y-axis shows the film's heat transfer coefficient, defined as [power dissipation / (film temperature - air temperature)]. Rotation Angle denotes wind direction, with $0^{\circ}$ signifying that film 1 is pointing directly into the wind. Velocities have been scaled to show equivalent velocities in carbon dioxide 635 at a temperature of $250 \mathrm{~K}$ and a pressure of 6 mbar.

Each curve shows data for one of the three films (averaged results from one clockwise and counterclockwise rotations). The $y$ axis expresses sensor performance as a heat transfer coefficient $C$ film $=$ $q_{\text {film }} /\left(T_{\text {film }}-T_{\text {air }}\right)$, where $q_{\text {film }}$ and $T_{\text {film }}$ represent respectively the heat dissipation (in $m W$ ) and 640 temperature (in K) of the hot film.

The convective part of the heat loss from each film is calculated simply by subtracting the heat flow experienced when no wind is present $C_{c o n v}=C_{t o t a l}-C_{(u=0)}$. This convective heat transfer coefficient is then converted to a dimensionless Nusselt number $N u=C d / A k$, where $d$ and $A$ represent the diameter and area of the film, and $k$ represents the thermal conductivity of the atmosphere. Finally, the baseline data analysis method uses the differences between the films to calculate wind speeds. 
646 From this we calculate an effective Nusselt vector $\left(N u_{x}, N u_{y}\right)$ from which a $2 \mathrm{D}$ wind vector is then calculated as described in Wilson 2003b.

The possible shadowing of MetWind by the full assembled MetMast structure was investigated in the Aarhus Martian wind tunnel in Aarhus. Results will be reported in a separate paper.

The calibration of the MetWind sensor is prone to some of the same issues common to all thermal anemometers: the films respond not just to changes in wind speed but to all thermal loads. Uncertainties in the exact thermal environment around the MetWind sensor on Mars, due for example to changing temperatures of lander structure near MetWind, would have resulted in significant uncertainties in the absolute calibration of MetWind. If one side of the wind sensor was heated by proximity to a hot portion of the lander, for example, that would produce the same response in MetWind as a wind blowing toward that direction; such asymmetrical thermal loads could cause errors of up to $4 \mathrm{~m} / \mathrm{s}$ in the measured wind vector in extreme cases, and up to $1 \mathrm{~m} / \mathrm{s}$ in nominal cases. Ultrasonic time-of-flight wind sensors, as used for turbulence study on Earth, would provide a more robust calibration and faster response time (10 Hz compared to $0.2-0.5 \mathrm{~Hz}$ for MetWind). But MetWind proved well suited for the DREAMS goals of providing a basic landing site climatology and first-order turbulence characterization in an extremely compact and robust form.

\subsubsection{MicroARES}

Given that MicroARES measures the potential of its electrode, the electric field values are derived from finite element modelling of the antenna and lander immersed in an atmosphere of conductivity

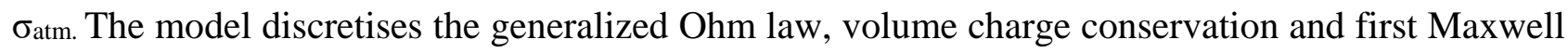
law equations ( $\left.\vec{E}=\sigma_{a t m} \cdot \vec{J} ; \partial \rho / \partial t+\vec{\nabla} \cdot \vec{J}=0 ; \vec{\nabla} \cdot \vec{E}=-\Delta \mathrm{V}=\rho / \varepsilon_{0}\right)$ in order to properly simulate both the electric field deformations around the instrument and the resulting current collected by the electrode. The parasitic input values of the instrument (detailed below) are included in the 


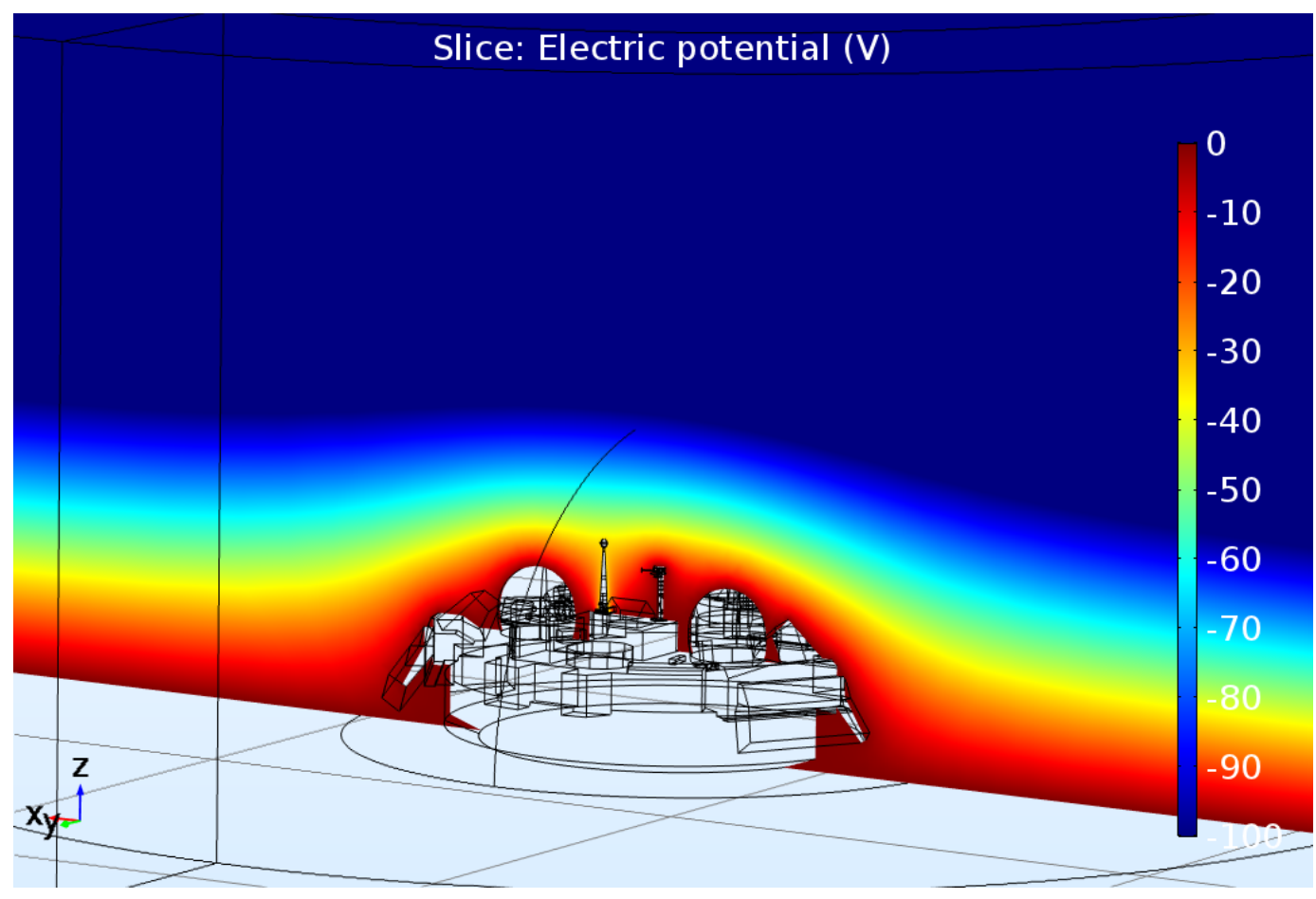

673 Figure 11: Electric field (-100V/m vertically constant) perturbations induced by the lander. The atmosphric conductivity is $2 \mathrm{pS} / \mathrm{m}$ and the resulting electrode potential measured by Micro-ARES is

In the calibration process (Déprez, 2016), signals are always injected in the instrument through socalled injection boxes supposed to reproduce the coupling of the electrode with the atmosphere (Berthelier et al., 2000). These boxes consist of a set of resistances and capacitors mounted in parallel and specified to have resistance and capacitance values close to conditions relevant for the MicroARES electrode when immersed in a Martian-like atmosphere.

To calibrate the various components of the instrument part, potential is measured at various test points and the necessary relations (linearity, gain or frequency response) are subsequently established. The measurement uncertainties are given in the datasheets of the various devices. However, when the test point of the instrument measurement is used (in Least Significant Bits, LSB) the value and error are respectively derived from the average and standard deviation of a measurement set. The uncertainties are then propagated by fitting the data with adequate functions, using the least-square method to take into account the uncertainties of all inputs. 
691 The DC chain was calibrated during Thermal vacuum tests, with temperatures ranging from $45^{\circ} \mathrm{C}$ to

692

693

694

695

696

697

698

699

700

701 $-35^{\circ} \mathrm{C}$ (every $5^{\circ} \mathrm{C}$ ). The DC linearity at each temperature with each Injection box and at 17 steps was measured between $-80 \mathrm{~V}$ and $+80 \mathrm{~V}$.

The electronic chain is described by equation (Millman theorem) shown below, which is fitted with data gathered during the TVT using the least-square method, in order to properly propagate the uncertainties:

$$
\begin{gathered}
V_{\text {injected }}(L S B, T, R e)=V_{\text {mes }}(L S B, T) \cdot\left(1+\frac{R e}{R i(T)}\right)-i L(T) \cdot R e \\
R i(T)=p_{4}+p_{5} \cdot T \\
i_{L}(T)=p_{6} \cdot \exp \left(p_{7} \cdot T\right)
\end{gathered}
$$

The resulting error on the computed electrode potential at $20^{\circ} \mathrm{C}$ is shown in Figure 12 .

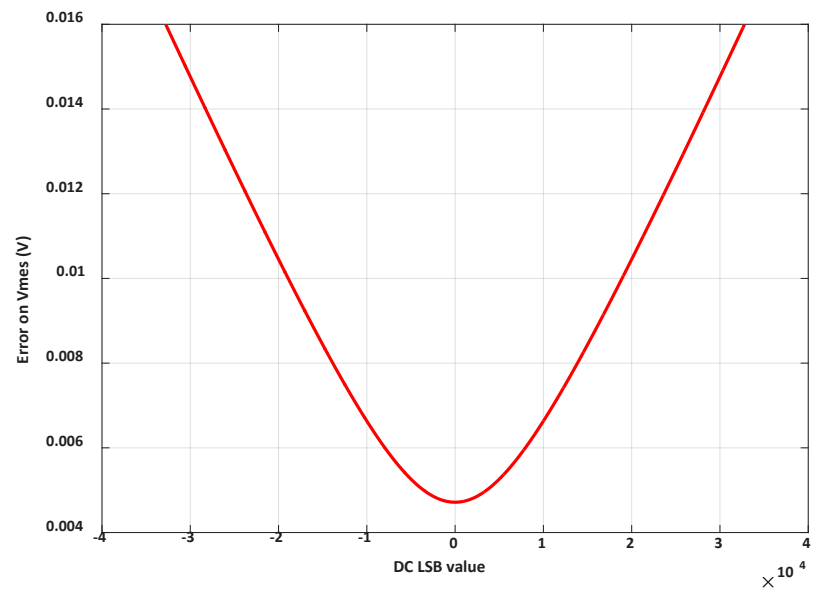

Figure 12: Error on the retrieved electrode potential at $20^{\circ} \mathrm{C}$

\section{AC Channel}

The AC channel calibration has been performed only at $20^{\circ} \mathrm{C}$, since the noise conditions and access to the instrument board during the TVT was limited.

Calibration consisted in measuring the frequency response, both in gain and phase, of the whole electronic circuit (Figure 13). 


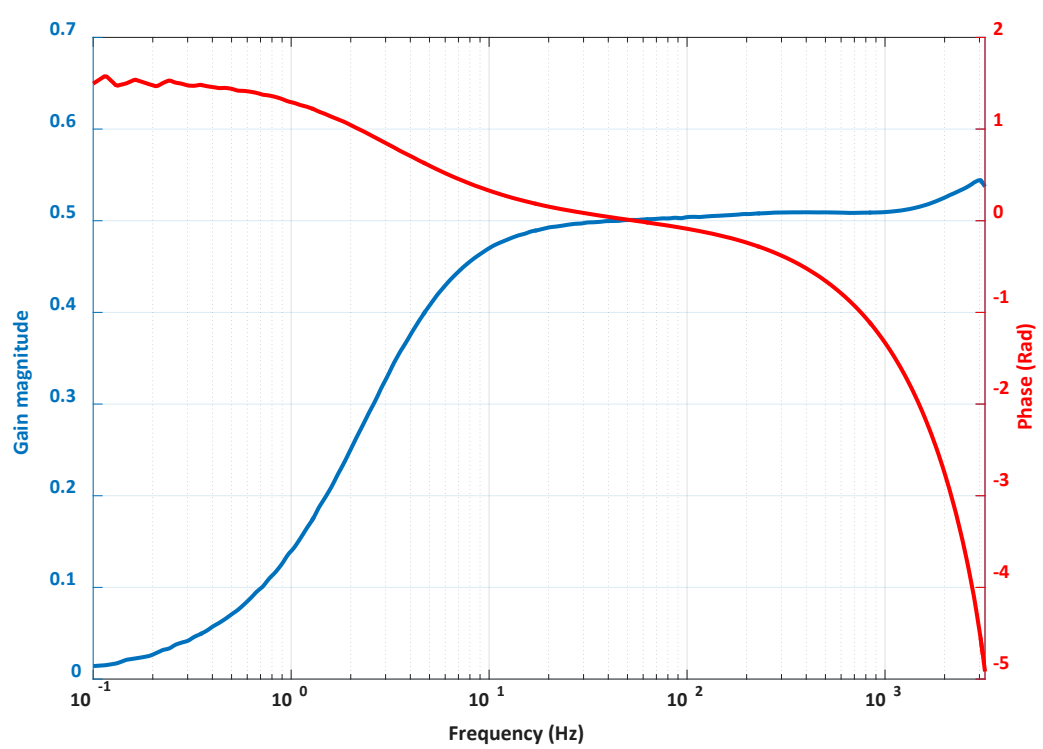

Figure 13: AC channel response in gain and phase

The calibration of SIS photodetectors consists of various steps. The first step is aimed at determining the responsivity to light normally incident to each detector. Then the variation of this response as the angle of incidence of light varies is measured to calibrate the angular response of each detector. Finally, the thermal dependence of the responsivity is measured, although the thermal effects were found to be very small.

The facilities used for this calibration are at INTA’s SPASOLAB (Space Solar Cell Test Laboratory, an official laboratory for solar cells testing according to ESA required standards). SPASOLAB has several sun simulators and Xenon lamps. A triple light source Sun simulator set to AM0 (Air Mass 0, i.e. outside the atmosphere) spectrum and a set of neutral filters are used for the normal-incidence responsivity calibration. For the angular dependence, $\mathrm{SIS} \mathrm{OH}$ is mounted on a rotating mechanism controlled by two high-precision $\left(0.01^{\circ}\right.$ step) motors that allows the relative azimuth and zenith angle between $\mathrm{OH}$ and the light source direction to be controlled. The thermal calibration is done in a thermal chamber with an optical quartz window. The accuracy of the lamp irradiance is known within $\pm 1 \%$. 
The dynamic range of the 3 different spectral channels is $1050 \mathrm{~W} / \mathrm{m}^{2}$ for the unfiltered (zenithpointing) detector, $390 \mathrm{~W} / \mathrm{m}^{2}$ for the NIR detectors, and $110 \mathrm{~W} / \mathrm{m}^{2}$ for the UV ones. The responsivities are $0.544 \mu \mathrm{A} /\left(\mathrm{W} / \mathrm{m}^{2}\right)$ for the unfiltered, around $1.38 \mu \mathrm{A} /\left(\mathrm{W} / \mathrm{m}^{2}\right)$ for the NIR detectors (with slight differences between them), and $0.202 \mu \mathrm{A} /\left(\mathrm{W} / \mathrm{m}^{2}\right)$ for $\mathrm{UV}$, with minor differences between the 3 UV detectors. The uncertainty (standard deviation) in the determination of these values ranges from $0.17 \%$ to $0.41 \%$, depending on the detector.

For the calibration of the angular dependence, the main source of uncertainty was due to the set-up itself. An error in the knowledge of the real angle of incidence of light of up to 0.5 degrees is assumed. This leads to errors in the accuracy of the angular dependence calibration of less than 5\% for \pm 30 degrees from the normal incidence. These errors rise up to $20 \%$ in the limit of the FoV, where the responsivity is less than $5 \%$ of that at normal incidence. Details can be found in Jiménez et al. (2016).

Thermal effects are small on SIS measurements, and become negligible after applying corrective factors as a function of the internal PT1000 sensor installed inside the $\mathrm{OH}$.

Finally, it is worth mentioning the small angular dependence of the unfiltered, zenith-pointed, detector. Its angular dependence shows revolution symmetry when varying azimuth, and regarding zenith angle, the responsivity is still higher than $90 \%$ of the normal incidence one for 35 degrees. It is still $80 \%$ of the normal incidence responsivity for a zenith angle of 50 degrees, whereas the total average responsivity is $81 \%$ of that for normal incidence, when its complete hemispherical FoV is considered. Thanks to this, to convert the measured photocurrent (A) into irradiance $\left(\mathrm{W} / \mathrm{m}^{2}\right)$, a direct application of the normal incidence responsivity value is possible, with acceptable error, regardless of the particular Sun position. Specially, if the Sun is in a low relative zenith angle, the direct, circumsolar, and most of the diffuse light will actually be received with a responsivity very close to the nominal (normal incidence) value, making the estimation of the absolute irradiance a very straightforward process. Figure 14, extracted form Jiménez et al. (2016), shows this angular dependence: 


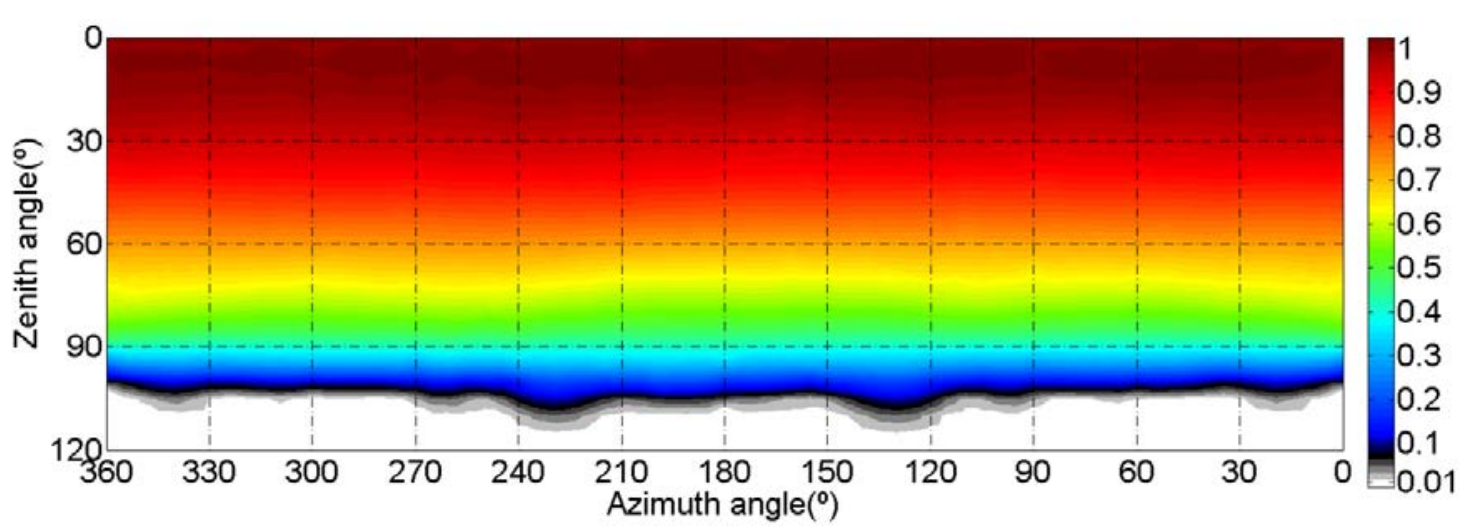

Figure 14: Angular dependence of the zenith-pointing SIS detector responsivity.

The performances of all SIS detectors are summarized in Table 2. During the trip to Mars, the accumulated radiation will degrade the detectors’ responsitivity in a different amount for each spectral band. Radiation analysis shows that the maximum signal degradation for the expected radiation levels (margins accounted) is $14 \%$ (for the NIR sensor which is the worst case). This can then be reduced thanks to the estimation of the Displacement Damage suffered by the detectors, obtained through the monitoring of the dark-current of the reference detector included for this purpose.

\begin{tabular}{|l|l|l|l|l|l|l|}
\hline \multirow{2}{*}{} & Incident light angle & $\begin{array}{l}\text { Noise, expressed as } \\
\text { equivalent } \\
\text { irradiance }\end{array}$ & \multicolumn{2}{l|}{ Accuracy* } & \multicolumn{2}{l|}{ Precision } \\
\hline & & {$\left[\mathrm{W} / \mathrm{m}^{2}\right]$} & Value & Unit & Value & Unit \\
\hline TOP & @ Normal incidence & $1.1 \cdot 10^{-3}$ & 1 & $\%$ & 0.04 & $\%$ \\
\hline & @ 50\% of the FoV & $2.2 \cdot 10^{-3}$ & 3 & $\%$ & 0.1 & $\%$ \\
\hline & Under diffuse light & $1.4 \cdot 10^{-3}$ & 1 & $\%$ & 0.003 & $\mathrm{~W} / \mathrm{m}^{2}$ \\
\hline NIR & @ Normal incident & $0.4 \cdot 10^{-3}$ & 1 & $\%$ & 0.004 & $\%$ \\
\hline & @ 50\% of the FoV & $0.8 \cdot 10^{-3}$ & 6 & $\%$ & 0.08 & $\%$ \\
\hline & @ 10\% of the FoV & $4 \cdot 10^{-3}$ & 10 & $\%$ & 0.4 & $\%$ \\
\hline & Under diffuse light & $0.5 \cdot 10^{-3}$ & 1 & $\%$ & 0.005 & $\mathrm{~W} / \mathrm{m}^{2}$ \\
\hline & @ Normal incident & $0.1 \cdot 10^{-3}$ & 2 & $\%$ & 0.04 & $\%$ \\
\hline & @ 50\% of the FoV & $0.2 \cdot 10^{-3}$ & 7 & $\%$ & 0.1 & $\%$ \\
\hline & @ 10\% of the FoV & $1 \cdot 10^{-3}$ & 10 & $\%$ & 0.4 & $\%$ \\
\hline & Under diffuse light & $0.12 \cdot 10^{-3}$ & 1 & $\%$ & 0.001 & $\mathrm{~W} / \mathrm{m}^{2}$ \\
\hline
\end{tabular}

Table 2: SIS performances as computed from calibration. Note: It must not be surprising that the indicated values in the table, even the noise, depend on the angle of incidence of the light. This is because the different magnitudes have been expressed as an equivalent irradiance. But to translate, for example, a noise, into irradiance, the sensor's calibration equation must be used. This equation includes an important parameter which is the Angular Response Function of the sensor, that depends on the angle of incidence of the light. For that reason the "equivalent irradiance" of that noise depends on the angle we may suppose in a hypothetical scenario. 
The calibration of the accelerometer inside the PE was done using precision motors and precision bubbles to fix the horizontal position for each axes. First the real orientation of each axis with regard to the PE box was found, and then rotations around the axis were applied in order to find the offset and sensitivity of each accelerometer. Only X and Y axes were acquired, allowing for a complete determination of the tilt angle of the XY plane after landing, and rotation of the $\mathrm{X}$ and $\mathrm{Y}$ axis within that plane. Different analysis were carried out to find the uncertainty that could be introduced by thermal effects and by the uncertainty in the knowledge of the gravity of Mars (which affects the calculation of the XY rotation within its plane). It was found that the required uncertainties in the temperature and gravity knowledge, needed to create a tilt uncertainty of $1^{\circ}$, were around $12^{\circ} \mathrm{C}$ and $1.5 \mathrm{~m} / \mathrm{s}^{2}$ respectively. The thermal effects were however calibrated by using a thermal chamber and some fixed inclinations provided by special support tools. The knowledge of gravity is better than $\pm 0.05 \mathrm{~m} / \mathrm{s}^{2}$.

The acquisition scheme of SIS makes use of an oversampling acquisition that provided around 20 bits of free-of-noise effective resolution for the photodetecting channels, as measured during the calibration campaign.

Finally, it is worth noting that, in a long-duration mission, dust deposition on the detectors surfaces will be the main source of degradation of the total responsivity. The use of magnets around the photodetectors, to slightly deflect the dust trajectory and thus minimize its deposition rate, was proposed for the Rover Environmental Monitoring Station (REMS) on Curiosity (Michael D. Smith et al., 2016). The same is being done in the Radiation and Dust Sensor (RDS) of MEDA, the nextgeneration meteorological station that INTA develops for the Mars2020 Rover (V. Apéstigue at al., 2015). Also, the responsivity degradation can be roughly estimated through the analysis of the yearly evolution of all the sensors lying in the same plane (a number of sensors, all of them showing similar long-term signal decrease), as experienced in REMS. 
Nevertheless, for all scientific retrieval algorithms for which relative instead of absolute signals are employed (i.e. the ratio between the signals delivered by detectors in different sides of the optical head), an in-situ calibration process is proposed. It is worth noting that the estimation of the optical depth can be done by means of these purely differential measurements. The method consists in comparing the signals provided by each couple of detectors when the Sun lies in a symmetric position with regard to their FoVs. In the absence of degradation, each detector should generate the same signal (within their initial differences in responsivity). This technique also allows, during the first Sols of a mission (when dust deposition is still negligible) to determine the orientation of the sensor after landing, as was demonstrated during the field campaign carried out in the Sahara desert (I. Arruego et al, 2017).

\subsubsection{DREAMS performances}

The performances of DREAMS sensors are summarized in Table 3.

\begin{tabular}{|c|c|c|c|c|}
\hline Sensor & Measured Quantity & Range & Resolution & Accuracy \\
\hline MarsTEM & Temperature & $70-320 \mathrm{~K}$ & $0.02 \mathrm{~K}$ & $0.1 \mathrm{~K}$ \\
\hline DREAMS-P & Pressure & 0-1015 hPa & $<0.5 \mathrm{~Pa}$ & $<5 \mathrm{~Pa}(\mathrm{BOL})\left(^{*}\right)$ \\
\hline DREAMS-H & Relative humidity & $0-100 \%$ & $0.5 \% \mathrm{RH}$ & $\begin{array}{l} \pm 10 \% \mathrm{RH} \text { down to }-70^{\circ} \mathrm{C} \\
\pm 20 \% \mathrm{RH} \text { down to }-83^{\circ} \mathrm{C} \\
+/-4 \% \mathrm{RH} \text { in dry conditions at } \\
\text { all T }\end{array}$ \\
\hline MetWind & $\begin{array}{l}\text { Wind speed } \\
\text { Wind direction }\end{array}$ & $\begin{array}{l}0.3-30 \mathrm{~m} / \mathrm{s} \text { and } \\
\text { above }\end{array}$ & $0.1 \mathrm{~m} / \mathrm{s}$ & $\begin{array}{l}+/-1 \mathrm{~m} / \mathrm{s} \\
+/-10^{\circ} \text { for wind speeds }>5 \\
\mathrm{~m} / \mathrm{s}\end{array}$ \\
\hline MicroARES & $\begin{array}{l}\text { Vertical Electric } \\
\text { Field and potential }\end{array}$ & $\begin{array}{l}\text { DC channel } \\
-256 \text { to }+256 \mathrm{~V} / \mathrm{m} \\
\text { in native mode } \\
>10 \mathrm{kV} / \mathrm{m} \text { in } \mathrm{HV} \\
\text { mode, depending } \\
\text { on the atmospheric } \\
\text { conductivity } \\
\text { AC channel }\end{array}$ & $\begin{array}{l}\text { DC channel } \\
8 \mathrm{mV} / \mathrm{m} \text { in native } \\
\text { mode } \\
\sim 0.1 \mathrm{~V} / \mathrm{m} \text { in } \mathrm{HV} \text { mode, } \\
\text { depending on the } \\
\text { atmospheric } \\
\text { conductivity } \\
\text { AC channel }\end{array}$ & $\begin{array}{l}\text { DC channel } \\
60 \mathrm{mV} / \mathrm{m} \text { in native mode. } \\
\sim 1.2 \mathrm{~V} / \mathrm{m} \text { in } \mathrm{HV} \text { mode. }\end{array}$ \\
\hline
\end{tabular}




\begin{tabular}{|c|c|c|c|c|}
\hline & & $\begin{array}{l}\text { 4-3200 } \mathrm{Hz} \\
\text { frequency range } \\
\\
15 \mathrm{~V} / \mathrm{m} \text { peak-to- } \\
\text { peak in standard } \\
\text { mode. } \\
0.2 \mathrm{~V} / \mathrm{m} \text { peak-to- } \\
\text { peak in high } \\
\text { sensitivity mode. }\end{array}$ & $\begin{array}{l}0.26,0.52 \text { and } 25 \mathrm{~Hz} \\
\text { spectral resolution in } \\
\text { the respective } 4-50 \text {, } \\
50-100 \text { and } 100- \\
3200 \mathrm{~Hz} \text { bands. } \\
0.2 \mathrm{mV} / \mathrm{m} \text { in standard } \\
\text { mode. } \\
3 \mu \mathrm{V} / \mathrm{m} \text { in high } \\
\text { sensitivity mode. }\end{array}$ & $\begin{array}{l}0.15 \mathrm{mV} / \mathrm{m} \text { in standard mode. } \\
2 \mu \mathrm{V} / \mathrm{m} \text { in high sensitivity } \\
\text { mode. }\end{array}$ \\
\hline SIS & $\begin{array}{l}\text { Solar Irradiance } \\
\text { Dust opacity } \\
\text { UV intensity }\end{array}$ & $\begin{array}{l}\text { Under direct } \\
\text { normal light: } \\
\text { Tot: } 220-1200 \mathrm{~nm} \text { : } \\
\text { 0-1050 W/m² } \\
\text { NIR: } 700-1100 \mathrm{~nm} \text { : } \\
\text { 0-390 W/m² } \\
\text { UVA: } 315-400 \mathrm{~nm}: \\
\text { 0-110 W/m² }\end{array}$ & $\begin{array}{l}\text { Under direct normal } \\
\text { light: } \\
\text { TOP: } 1 \mathrm{~mW} / \mathrm{m} 2 \\
\text { NIR: } 0.4 \mathrm{~mW} / \mathrm{m} 2 \\
\text { UV: } 0.1 \mathrm{~mW} / \mathrm{m} 2\end{array}$ & $\begin{array}{l}\text { Depends on the angle of } \\
\text { incidence of the light: } \\
\text { Tot. irrad.: } 1-30 \% \text { (30\% for } \\
\text { SZA }>85 \mathrm{deg}) \\
\text { NIR: } 1-10 \%\end{array}$ \\
\hline
\end{tabular}

811 Table 3: Performances of the DREAMS sensors. $(*)$ The reported value of the accuracy of the 812 pressure sensor refers to channel 2 of both DREAMS-P1 and DREAMS-P2 sensors (see Sect.

813 3.1.2).

\subsection{In-flight performances}

816 During the trip to Mars all the DREAMS sensors were activated for calibration and health check

817 tests. Sensors have been activated for three nominal and two additional checkouts between April

818 and September 2016. All sensors performed nominally. 

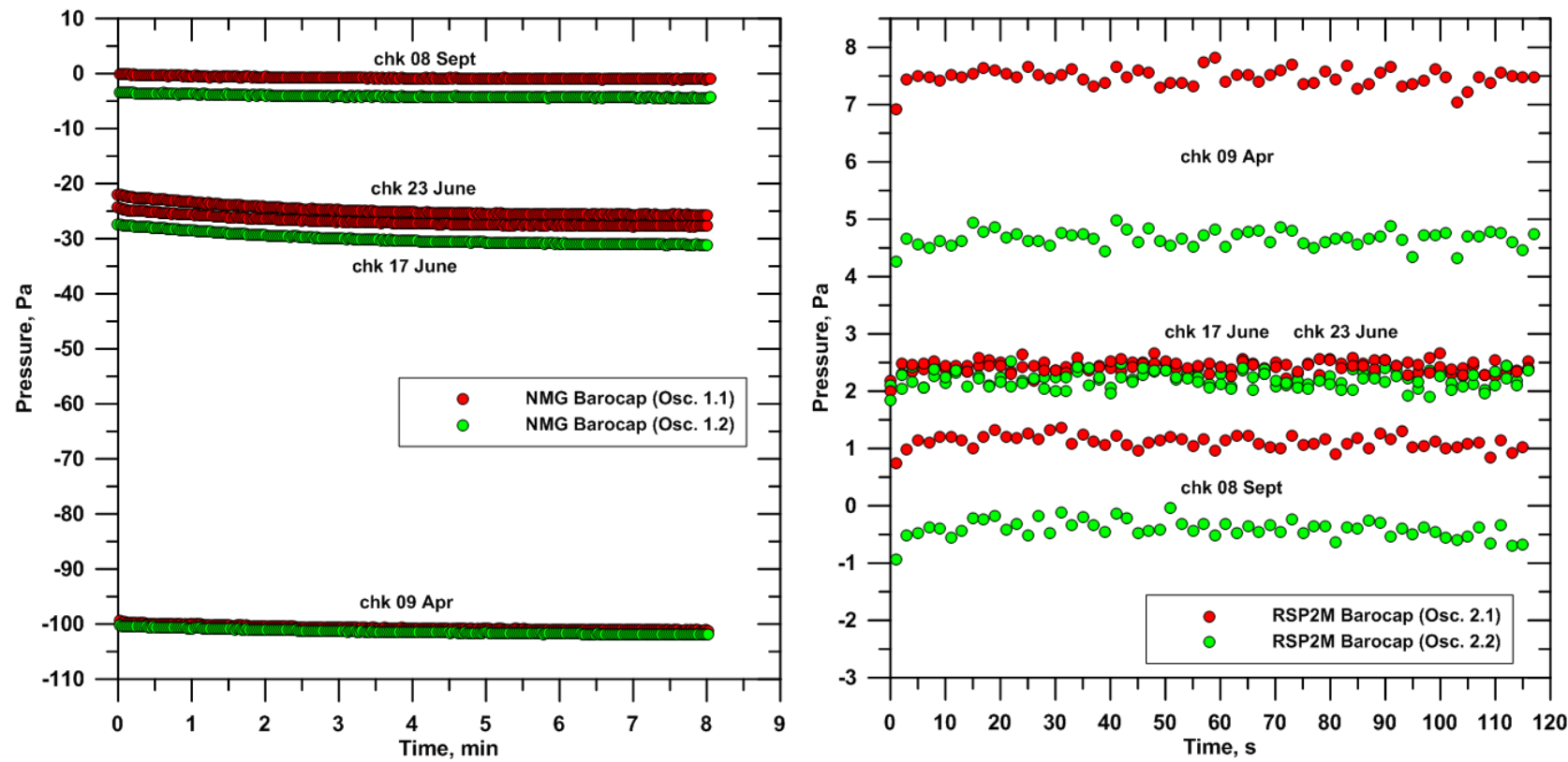

Figure 15: DREAMS-P pressure reading during the cruise checkouts on $9^{\text {th }}$ April, $17^{\text {th }}$ and $23^{\text {rd }}$ June and $8^{\text {th }}$ September, respectively. Readings from channel 1 of each pressure sensor are represented in red, while green indicates data from channel 2. P1 sensor (left plot) shows untypical behaviour going from -100 Pa reading at the first checkout to about $0 \mathrm{~Pa}$ in September. Note that P1 sensor has no space flight heritage. P2 sensor behavior is as expected.

During the cruise, Schiaparelli was encapsulated inside the spacecraft rear and front shields.

Vacuum and dry conditions were expected inside the spacecraft. Thus, the readings from

DREAMS-P and DREAMS-H have been used to correct on-ground calibration for the natural drifting of sensors and other effects. Figure 15 shows the trend of P1 and P2 sensors readings during the various checkouts. As expected, both sensors experienced outgassing during the cruise. The trend of P1 sensor resulted untypical but both sensors resulted completely outgassed and with reading of few $\mathrm{Pa}$ about one month before the landing.

DREAMS-H offset was also measured during all the checkouts.

In the last checkout before landing phase it was between $-2.4 \%$ and $-1.5 \%$ depending on Humicap sensor (Figure 16). 

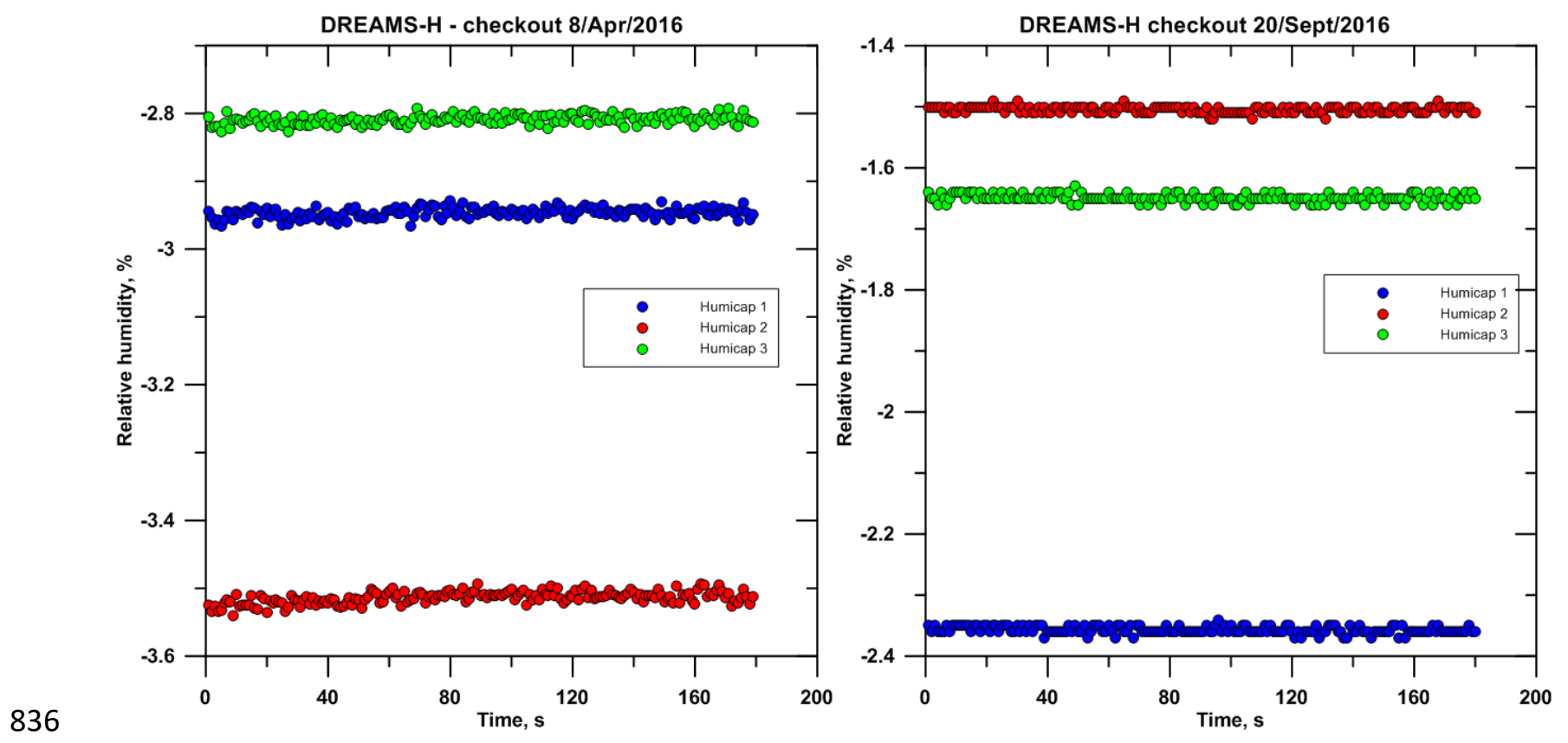

837 Figure 16: DREAMS-H reading during the first (left) and last (right) checkout.

MarsTEM behavior was nominal (Figure 17).

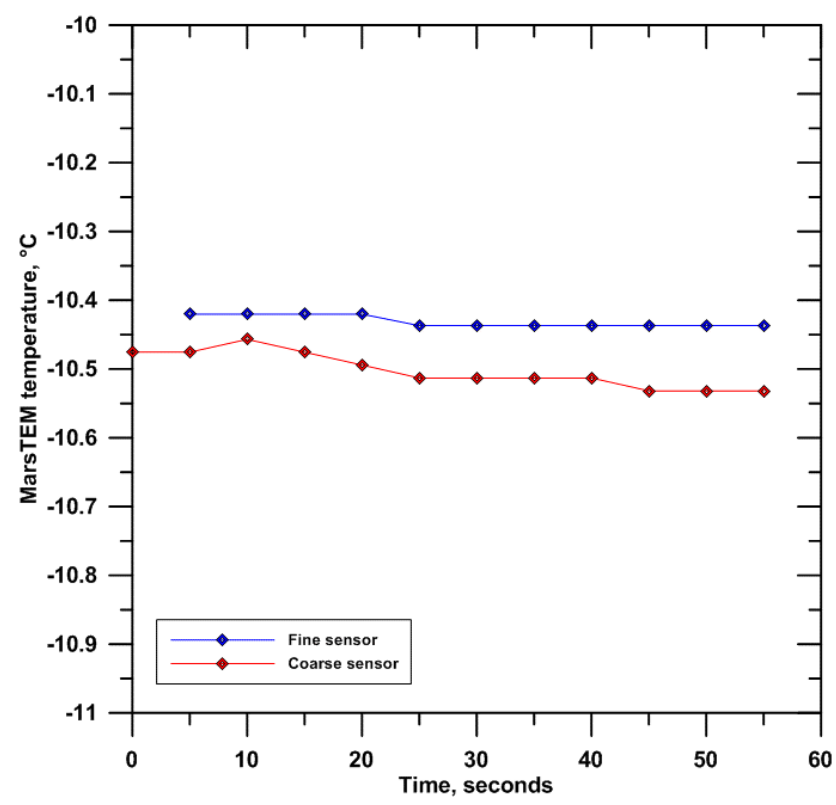

841 Figure 17: MarsTEM signal as acquired during the June checkout.

MetWind was turned on several times during cruise. Figure 18 shows the evolution of film temperatures during the last in-cruise check-out, on September 2016. The plot shows the 
the MetWind sensor, as described in Section 2.4. During the first 30 seconds, the sensor was in its low power mode where only $~ 2 \mathrm{~mW}$ are dissipated in the films. After 30 seconds MetWind was switched into its wind sensing mode, where $\sim 40 \mathrm{~mW}$ are dissipated in the films, to check the correct functioning of its electronics. This high power mode was used only for ten seconds to avoid overheating of the films, a condition which can arise in the vacuum of space when the films are not convectively cooled. The behavior of the wind sensor in these tests was nominal.

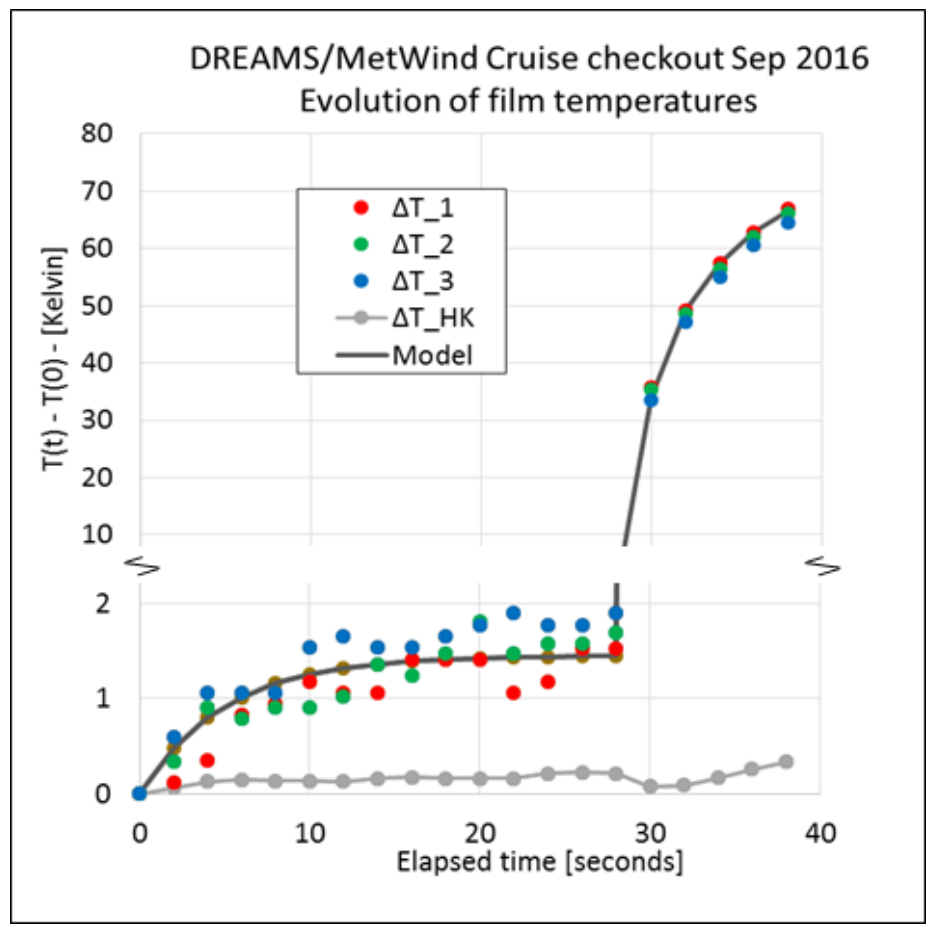

Figure 18: MetWind film temperatures during the last in-flight checkout, in Sep 2016. Heating power in the films was $\sim 2 \mathrm{~mW}$ for the first 30 seconds, $40 \mathrm{~mW}$ for the subsequent 10 seconds. Note the discontinuity in the vertical scale. $\Delta \mathrm{T}$ _HK refers to a housekeeping temperature, measured at the centre of the MetWind sensor.

MicroARES health check produced also expected results in terms of housekeeping values and sensor behavior.

SIS confirmed the excellent quality of the signals. Figure 19 shows some of the telemetry acquired during the last activation before reaching Mars. 

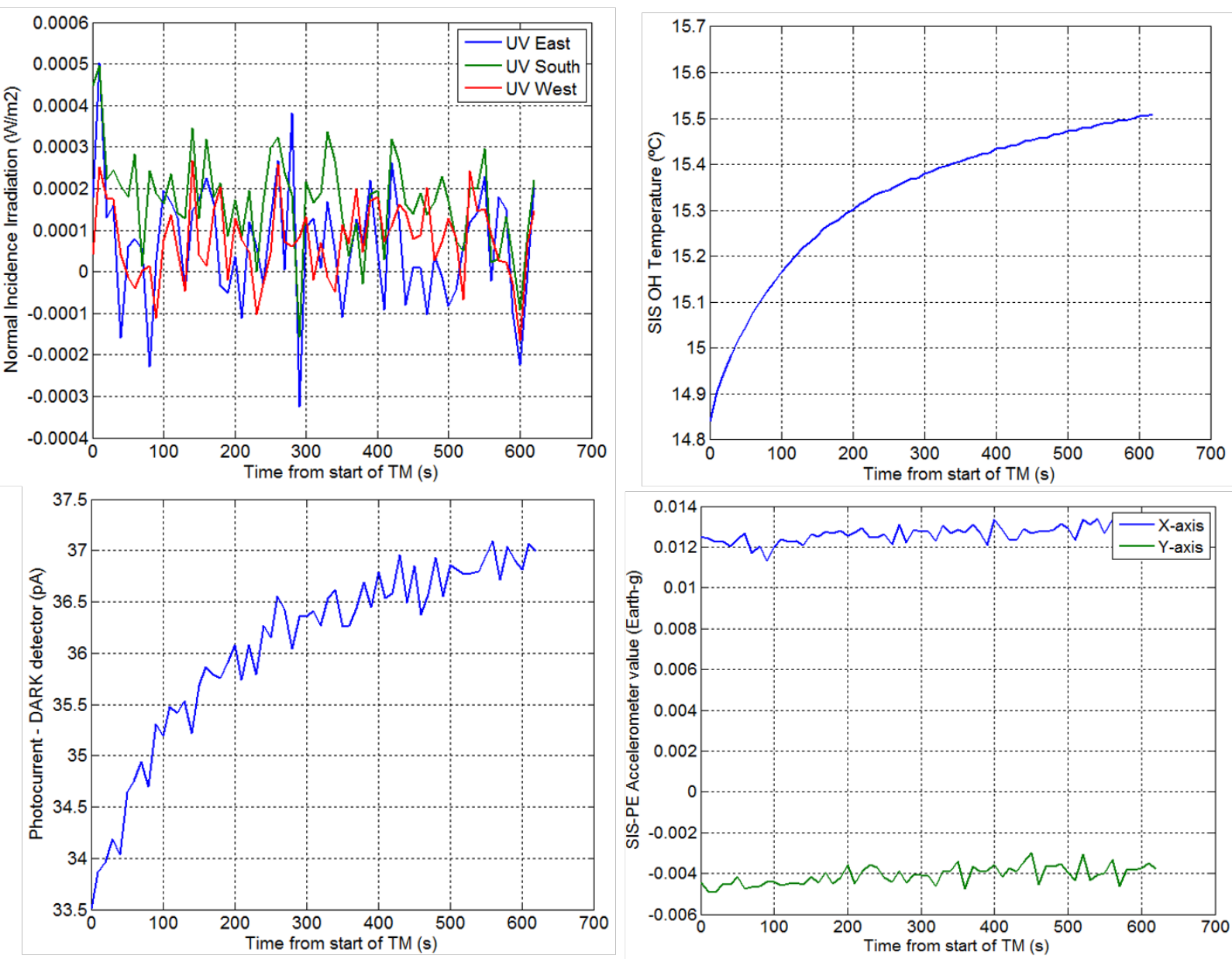

Figure 19: Some telemetry from the SIS activation on September $21^{\text {st }}$. From top to bottom and left to right: signals from the UV detectors expressed as the equivalent normal-incidence irradiance that would be necessary to generate the measured photocurrents, $\mathrm{OH}$ temperature, Dark current of the

As it can be seen, the noise in the UV photodetectors show a standard deviation equivalent (when converted to irradiance) to around $0.1 \mathrm{~mW} / \mathrm{m}^{2}$, which compared to the $110 \mathrm{~W} / \mathrm{m}^{2}$ dynamic range equals $0.9 \cdot 10^{-6}$ (i.e. more than 20 bits of free-of-noise resolution). The noisiest channel, due to the high gain employed in the current-to-voltage conversion, was the dark-current one. For this channel,

872 the requirement was to measure from pA to almost 40nA (which would be equivalent to more than 87315 bits of free-of-noise resolution). The telemetry shows that the pA resolution was actually 874 achieved. 
876 The DREAMS in-flight data are archived into the European Space Agency's Planetary Science

877 Archive (Schipani et al., 2016). The archive adopts the NASA's Planetary Data System version 4 878 (PDS4) standards as a baseline for the formatting and structure of all data.

879 


\section{Test in the Sahara desert}

881 DREAMS was fabricated to land on Mars during the dust storm season. For this reason, the instrument has been designed to cope with a dusty environment. The landing during the dust storm season would also provide a great opportunity to study, for the first time, the effect of dust on the electric properties of the Martian atmosphere.

Field experiments have been performed in the Sahara desert in 2013 and 2014, during the dust storm season, to test sensor response to harsh environment and study the relation between the emission of dust and the electric properties of the atmosphere. The results of these tests are a good start point to understand the dusty environment of Mars from future DREAMS acquired data.

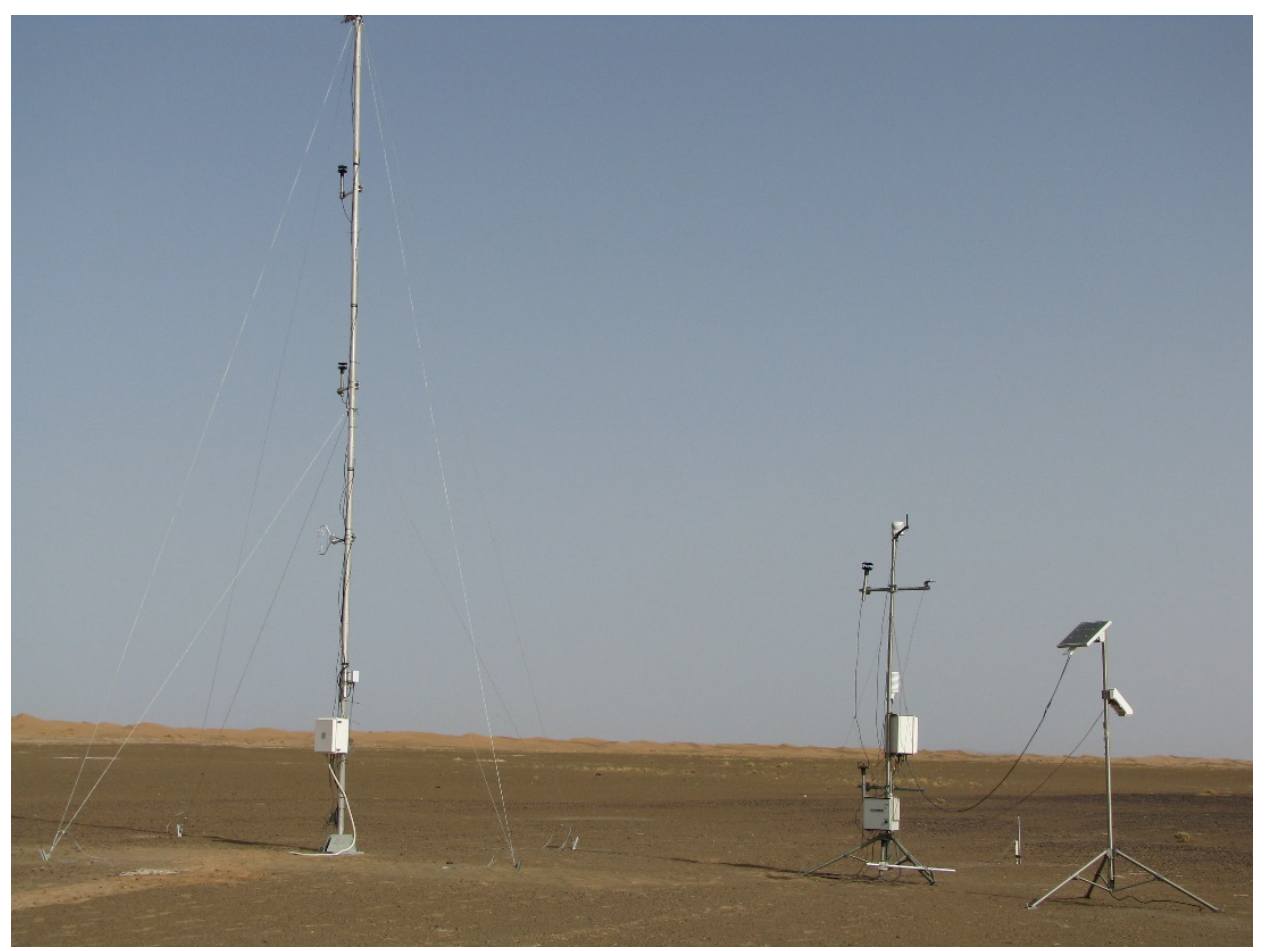

Figure 20: Meteorological station deployed in the Sahara desert for the study of dust lifting process

The scientific goal of the field campaign was the study of the development and evolution of dust storms and dust devils. For this aim, a meteorological station (see Figure 20), particularly devoted to the monitoring of sand saltation process (the bouncing of sand grains above the soil when blown by the wind) and dust entrainment in the atmosphere, has been deployed in the region around Merzouga 
897 (Errachidia) in the Moroccan desert. This desert has a Martian-like terrain and offers a good chance 898 to understand the physics behind aeolian processes. The chosen area is particularly rich in both sand 899 and dust particles and it is very active from the aeolian point of view.

900 The following physical quantities have been monitored for six months during the Summers 2013 and 901 2014: wind speed and direction at six different altitudes (wind vertical profile), atmospheric humidity, 902 temperature at two heights, pressure, solar irradiance, soil moisture and temperature, sand saltation 903 rate (by detecting the impacts of sand particles over two impact sensors), atmospheric electric field 904 (Campbell CS110) and the size distribution and number density of dust grains entering into the 905 atmosphere. The goal was to correlate environmental parameters to sand movements and dust 906 entrainment and, in particular, verify the effect of sand saltation process on the 907 generation/enhancement of the atmospheric electric field. Data analysis shows that charging of grains 908 during aeolian processes produces an enhancement of the atmospheric electric field. E-fields up to 20 $909 \mathrm{kV} / \mathrm{m}$ have been observed during the most intense dust storms. Note that the fair weather field is of 910 the order of 50-100 V/m. The field intensity results to be linearly related to the number of lifted grains 911 and negatively correlated with relative humidity. Moreover, data indicate that strong E-fields are also 912 capable to enhance dust lifting in a feedback process. A detailed discussion of campaign results is 913 reported in Esposito et al. (2016). Similar data could be collected by DREAMS instrument on Mars. 914 During July 2014, some of the DREAMS sensors, MicroARES, MarsTEM and SIS, were added to 915 the meteorological station deployed in the desert (Figure 21). The goal was to test their behavior 916 during dusty events. 

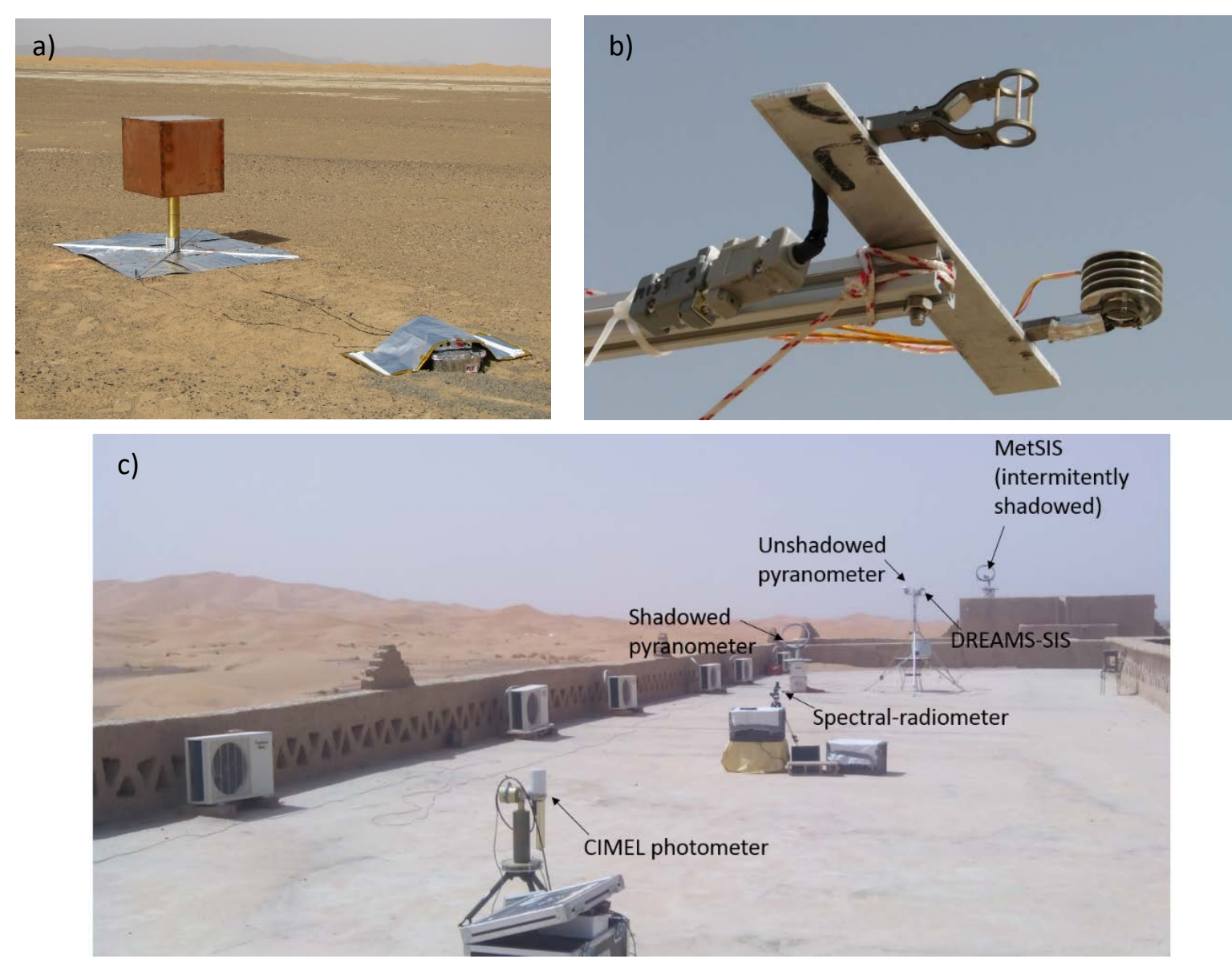

Figure 21: DREAMS sensors added to the meteorological station deployed in the Moroccan desert: a) Terrestrial version of MicroARES; b) At the top: the MarsTEM temperature sensor without the radiation shield; on the bottom right: the MarsTEM sensor protected by the aluminium shield; on top, fixed to the shield, the PT100 B sensor and in the shadow of the shield the PT100 A, directly exposed to air; c) SIS and other devices.

We used a terrestrial version of MicroARES antenna that was larger than the original one (Figure 21 a). This was due to the different electric conductivity on Earth and Mars. For atmospheric conductivity roughly 100 times smaller than that of Mars, an electrode roughly 100 times larger is needed in order to keep the sheath resistance Rs at the same order of magnitude. A greater Rs would cause the electrode not to be able to maintain itself at the floating local potential because of the small bias current flow to the input amplifier. The terrestrial model was a $60 \mathrm{~cm}$ side copper cube. Its 2.16 $\mathrm{m}^{2}$ surface area was equivalent to a $41.6 \mathrm{~cm}$ radius sphere, around 30 times larger than the original antenna. For more details see Harrison et al. (2016). 
Given the fact that the Campbell CS110 electric field mill mounted on the meteorological station can only measure electric field signals with a frequency below $0.5 \mathrm{~Hz}$, only the DC data can be compared to MicroARES sensor. However, the two instruments were located 50 meters apart, with a setup at 2 meters from the ground for the CS110, and a setup at $50 \mathrm{~cm}$ from the ground for MicroARES. Therefore, a meaningful comparison can only remain at a qualitative level.

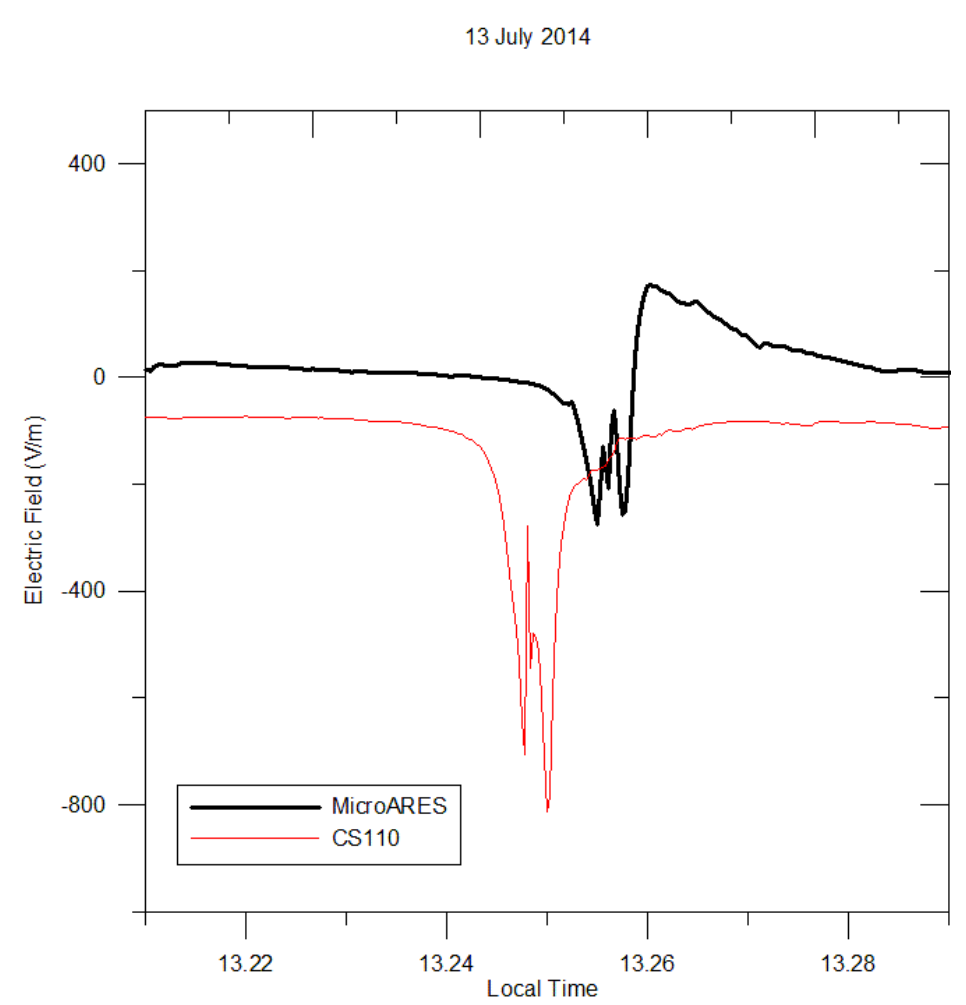

Figure 22: Dust-devil cross detection by CS110 electric field mill and Micro-ARES.

The ability of Micro-ARES to provide interesting data is confirmed by the concomitant dust devil detection made by the two instruments on many occasions (one example is shown in Figure 22). The signatures of the dust devil exhibit expected differences but overall provide excellent agreement with regard to the shape and timing of the sudden electric field rises and demises.

Two prototypes of the MarsTEM sensor were tested and compared during three days of measurements: a Titanium sensor structure with an aluminium shield and a free-shield sensor with a more complex structure made with Titanium alloy and peek rods (Figure 21 b). Several reference 
sensors (PT100) were positioned on the upper and lower part of the shield for comparison. During the testing the weather was variable, clouds were observed both in daytime and in night-time, wind changed both in velocity and direction and relative humidity changed in the range $(0 \ldots+50 \%)$. Some rainy events occurred lasting around half an hour, and some very weak dust storms were detected by the instruments. Temperature data were sampled at a $1 \mathrm{~Hz}$ frequency.

Daytime data showed clearly that the aluminium shield heated up and the temperature measured by the MarsTEM sensor was affected by a non negligible bias. For this reason, and because of similar effect foreseen by an ad hoc developed simulation using a CFD code in a Martian environment (see Chiodini et al. 2015), it was decided to not equip the MarsTEM with the aluminium protecting shield. During the nights, measurements were conducted continuously and data registered on the data logger; several peculiar events were observed and analysed (see Colombatti et al 2015). Evidence of a strong correlation of temperature, wind (both for velocity and direction), humidity and topography was highlighted.

A test campaign was also carried out with SIS (Figure 21 c). Different procedures for estimating the atmospheric optical depth (OD) were applied. One of them is based on fitting the measured photocurrents to the expected ones according to simulations made with radiative transfer models for different atmospheric conditions. The second, is based on the same fitting but working with signals (and model estimations) normalized to their own maximum, i.e., paying attention to the shape of the signals but not to their absolute values (thus rendering negligible any possible dust accumulation effect). The last procedure compares the photocurrents generated, at each moment, by the one of the three equal lateral detectors that has the Sun inside its FoV, and the sum of the two others. In other words, it compares global (direct plus diffuse) and diffuse light. The three procedures are detailed in Arruego et al. (2017), whereas the radiative-transfer model employed to simulate the SIS signals is described in Toledo et al. (2017). From the set of OD estimations obtained with the three different methods outlined, and with the different lateral detectors (a fitting 
between each detector's signal and the model, was done), the standard deviation of the estimations was found to be less than $18 \%$.

Regarding the absolute irradiance measured with the zenith-pointing detector, it was compared to that provided by a set of two pyranometers (un-shadowed one for global light measurement, and shadowed one for diffuse light measurement). The difference between the two estimations (SIS and pyranometers) was well below $10 \%$ for solar zenith angles (SZA) as high as $50^{\circ}$ (Arruego et al., 2017).

All tests confirm that DREAMS sensors perform nominally in harsh environment and are absolutely well suited for the monitoring of environmental parameters during dust events.

\section{Operations}

Surface operation timeline

DREAMS lifetime is limited by the capacity of its power unit and the power profile during operations. The acquisition sequence was designed as a compromise among scientific goals, available energy and communication constraints due to the sequence of orbiters passages over the landing site.

In order to cope with the science goals, measurements sequence has been built in order to:

- Be almost periodic all along the day and night time. This allows to monitor the diurnal cycle of atmospheric parameters.

- Make them more frequent in the daytime and longer at midday. Indeed, the Martian climate database (http://www-mars.lmd.jussieu.fr/) predicts a peak in surface temperature around 13:00 Mars local true solar time (LTST) in the landing site and period (Bertrand et al., 2016). Most of the convective activity, like high-frequency fluctuations of atmospheric 
wind, temperature, pressure, is expected roughly between 11:00 and 15:00. This is the most favorable period to get stronger winds and dust events (devils, storms).

- Have a long duration measurement at twilight in order to capture the highest relative humidity of the night (at sunset) and study cloud properties.

To fulfill the scientific objectives DREAMS is able to control each sensor independently, setting the start and stop time of each acquisition sequence, the acquisition rate and other parameters needed to configure the internal sensors (namely Sensor SWitch sequence: SSW). The unit is able to store in its non-volatile memory six different operation timelines. Each timeline is a list of SSW sequences to be executed in different phases of the mission. Two fail-safe mission timelines are hard-coded in the application software and cannot be changed, while the other four timelines are used for the nominal mission and can be uploaded to the unit during the interplanetary cruise to Mars. One of them is used to support the in-flight checkout operations. The other three timelines are used to implement the surface phase of the mission, they are called MTL1, MTL2 and MTL3 for convenience. Each timeline covers the acquisitions foreseen during one Martian sol starting from the local midnight. The acquisition sequences are triggered with respect to the Mars LTST computed at the center of the landing ellipse. In this way the acquisitions are correlated with the local events e.g. sunrise, sunset and specific part of the sol.

DREAMS is designed to operate autonomously once on the Mars surface. After the touch-down the descent module powers-on DREAMS, provides the mission time to the unit and then sets DREAMS in surface mode. Once in surface mode DREAMS starts executing the MTL1, then MTL2 and MTL3. At the beginning of the fourth sol DREAMS will execute again the stored MTLs starting from MTL1 and so on untill the depletion of its internal battery. Acquired data is stored in the nonvolatile memory of the unit waiting for the upload to the descent module. To save energy both DREAMS and the descent module are able to enter a low-power mode. The two units are kept synchronized with their internal timers and wake-up simultaneously. At each wake-up DREAMS 
uploads data to the descent module, then the descent module uploads data to the orbiters, notifies to DREAMS the time of the next communication window and enters hibernation again.

The design and validation of the three surface mission timelines is a crucial step for the success of the mission. These timelines are designed to satisfy several conditions listed below:

- Guarantee at least 2 sols of operation

- Max energy consumption of 120 Wh with 5\% margin at the last data upload

- Acquisition and storage of at least 50 Mbit of data for each sol

- Guarantee that DREAMS is ready to wake-up when expected by the descent module (with no more than 500 ms of margin)

- Guarantee that during the communication with the descent module, MicroARES is off to avoid disturbing the UHF link with the orbiters.

The final timeline is then tested simulating a two sols mission on the DREAMS Flight Spare model. The final surface mission timelines uploaded to DREAMS are depicted in Figure 23. The touch down was expected on 2016-10-19 at 14:48:17. Some seconds after the touch down the initialization of the DREAMS takes place and the first hibernation cycle begins. The first SSW to be executed is the SSW \#19 of the MTL \#1 (first sol of operation). The nominal mission foresees the execution of 43 SSWs listed in Table 3. These SSW correspond to about 11 hours and 20 minutes of data acquisition over 51 hours and 48 minutes of operation (see green markers in Figure 23). During each SSW all the sensors are acquired simultaneously with different configurations depending on the local time. The overall data volume uploaded to the descent module is about 118 Mbit over 2 sols. The internal battery depletion was expected around 2016-10-21 at 18:50:00. Nevertheless, the MTLs contains additional SSWs (gray markers) that could be executed in an extended mission. The energy stored in the battery depends on the thermal conditions experienced by the instrument during the cruise and at the surface, hence a warmer environment could result in more energy available for the experiment. 


\begin{tabular}{|c|c|c|c|c|c|}
\hline MTL & ssw & UTC & Local time & Time [s] & Data [Mbit] \\
\hline \multirow[t]{10}{*}{1} & 19 & 20161019T15:12:20.000 & 13:40:00.808 & 1528 & 6.559 \\
\hline & 20 & 20161019T15:53:27.000 & 14:20:00.773 & 1530 & 6.616 \\
\hline & 21 & 20161019T17:10:32.000 & $15: 35: 00.100$ & 1117 & 3.097 \\
\hline & 22 & 20161019T17:41:23.000 & 16:05:00.803 & 406 & 1.057 \\
\hline & 23 & 20161019T18:07:04.000 & 16:29:59.930 & 762 & 2.178 \\
\hline & 24 & 20161019T18:48:11.000 & 17:09:59.895 & 406 & 1.057 \\
\hline & 25 & 20161019T19:31:22.000 & 17:52:00.489 & 2182 & 5.527 \\
\hline & 26 & 20161019T20:25:51.000 & $18: 45: 00.660$ & 393 & 0.211 \\
\hline & 27 & 20161019T22:13:47.000 & $20: 30: 00.686$ & 393 & 0.163 \\
\hline & 28 & 20161020T00:17:08.000 & $22: 30: 00.574$ & 393 & 0.163 \\
\hline \multirow[t]{27}{*}{2} & 1 & 20161020T02:20:30.000 & 00:30:01.432 & 393 & 0.171 \\
\hline & 2 & 20161020T04:23:51.000 & 02:30:01.316 & 393 & 0.163 \\
\hline & 3 & 20161020T05:56:21.000 & 04:00:00.497 & 395 & 0.325 \\
\hline & 4 & 20161020T06:58:02.000 & 05:00:00.923 & 393 & 0.211 \\
\hline & 5 & 20161020T07:28:52.000 & $05: 30: 00.650$ & 2905 & 7.470 \\
\hline & 6 & 20161020T08:56:15.000 & 06:55:01.171 & 393 & 0.211 \\
\hline & 7 & 20161020T09:47:38.000 & 07:45:00.390 & 393 & 0.171 \\
\hline & 8 & 20161020T10:13:20.000 & 08:10:00.485 & 735 & 0.382 \\
\hline & 9 & 20161020T10:44:11.000 & 08:40:01.184 & 393 & 0.171 \\
\hline & 10 & 20161020T11:15:01.000 & 09:10:00.909 & 393 & 0.171 \\
\hline & 11 & 20161020T11:45:51.000 & 09:40:00.635 & 393 & 0.171 \\
\hline & 12 & 20161020T12:06:25.000 & 10:00:01.100 & 767 & 2.455 \\
\hline & 13 & 20161020T12:42:23.000 & 10:35:00.455 & 408 & 1.195 \\
\hline & 14 & 20161020T13:08:05.000 & 11:00:00.550 & 767 & 2.455 \\
\hline & 15 & 20161020T13:44:04.000 & 11:35:00.877 & 408 & 1.195 \\
\hline & 16 & 20161020T14:09:46.000 & 12:00:00.972 & 767 & 2.455 \\
\hline & 17 & 20161020T14:40:36.000 & $12: 30: 00.696$ & 1552 & 7.559 \\
\hline & 18 & 20161020T15:11:26.000 & 13:00:00.421 & 1552 & 7.559 \\
\hline & 19 & 20161020T15:42:17.000 & 13:30:01.118 & 1552 & 7.559 \\
\hline & 20 & 20161020T16:18:15.000 & 14:05:00.471 & 1127 & 3.633 \\
\hline & 21 & 20161020T17:40:29.000 & $15: 25: 00.383$ & 1127 & 3.633 \\
\hline & 22 & 20161020T18:06:11.000 & 15:50:00.477 & 1127 & 3.633 \\
\hline & 23 & 20161020T18:47:18.000 & 16:30:00.432 & 769 & 2.544 \\
\hline & 24 & 20161020T19:18:08.000 & $17: 00: 00.155$ & 408 & 1.244 \\
\hline & 25 & 20161020T20:11:36.000 & 17:52:00.972 & 2096 & 0.813 \\
\hline & 26 & 20161020T20:57:51.000 & 18:37:00.556 & 408 & 1.195 \\
\hline & 27 & 20161020T21:42:03.000 & $19: 20: 00.483$ & 408 & 1.195 \\
\hline \multirow[t]{6}{*}{3} & 1 & 20161021T04:02:23.000 & 01:30:00.290 & 407 & 1.130 \\
\hline & 2 & 20161021T08:03:57.000 & 05:25:00.367 & 2892 & 6.958 \\
\hline & 3 & 20161021T15:20:49.000 & 12:29:59.946 & 1484 & 4.527 \\
\hline & 4 & 20161021T15:51:40.000 & 13:00:00.636 & 1484 & 4.527 \\
\hline & 5 & 20161021T17:49:52.000 & $14: 54: 59.875$ & 1546 & 7.315 \\
\hline & 6 & 20161021T18:20:43.000 & $15: 25: 00.565$ & 1546 & 7.315 \\
\hline
\end{tabular}

1052 Table 3: sensor acquisition sequences foreseen during the nominal DREAMS mission. 

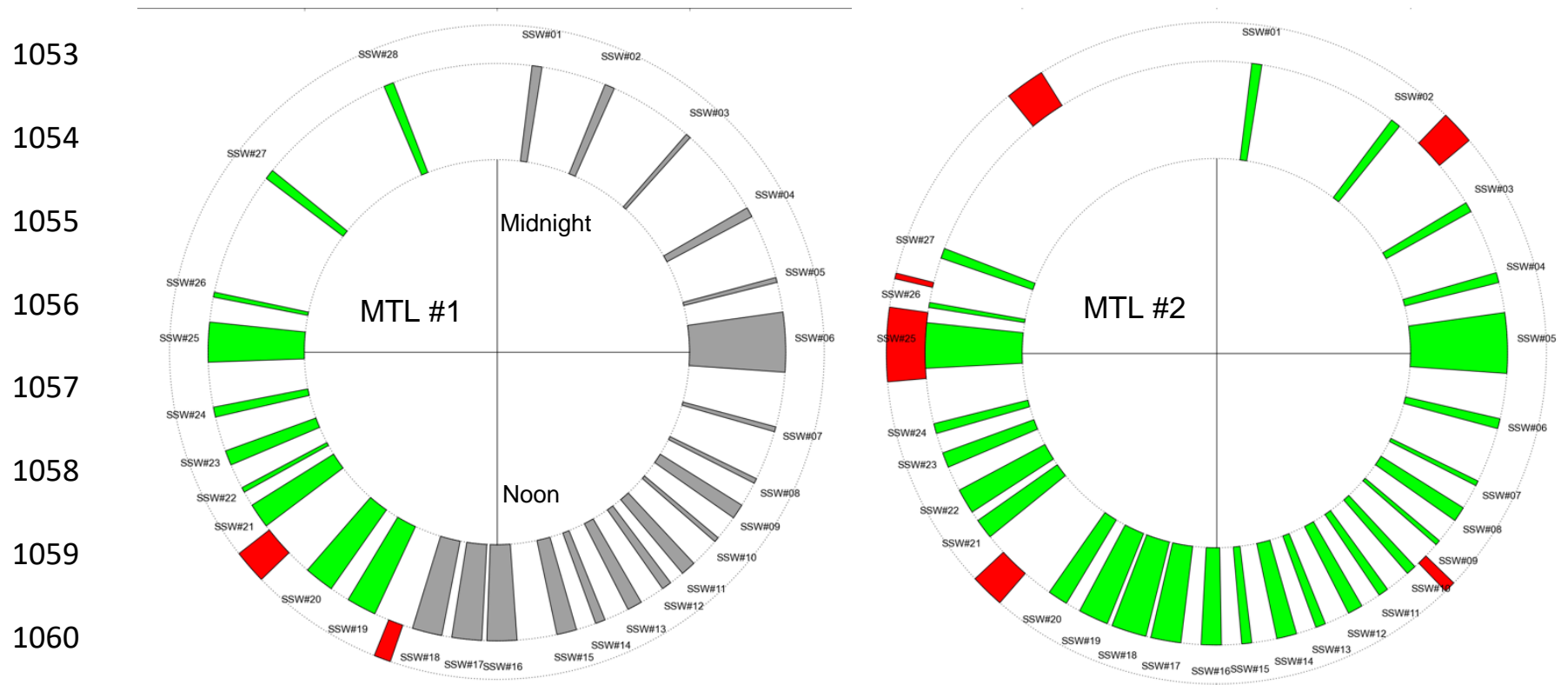

1061

1062

Touch down expected around

2016-10-19 at 14:48:17

End of mission expected around

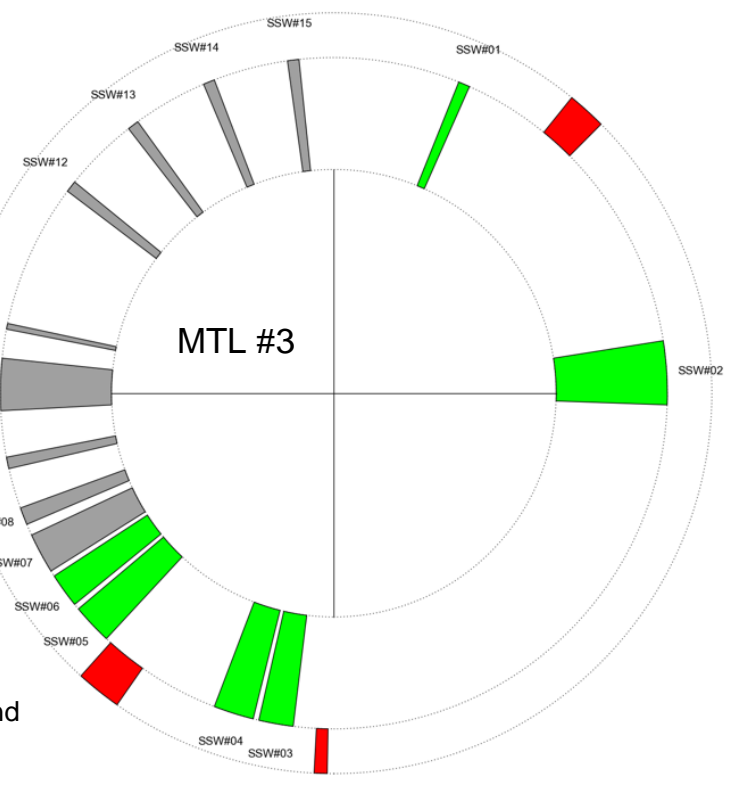

1069

2016-10-21 at 18:50:00

1070

Figure 23: Final surface timeline. Gray markers are the sensor acquisitions not executed, green are 1071 executed, while red markers are the communication windows with the descent module. 


\section{Conclusions}

1077 The Schiaparelli module of the ExoMars 2016 mission accommodated the DREAMS (Dust 1078 Characterization, Risk Assessment and Environmental Analyzer on the Martian Surface) experiment. 1079 DREAMS is a small meteorological station with the capability to measure electrical fields at the 1080 Martian surface. It includes six sensors: MarsTEM (thermometer), MetWind (anemometer), 1081 DREAMS-P (pressure sensor), DREAMS-H (humidity sensor), SIS (Solar Irradiance Sensor) and 1082 MicroARES (electric field sensor), a Common Electronic Unit and a Battery. It can operate 1083 autonomously according to a Mission Time Line and provide environmental measurements at the 1084 surface of Mars, including the first ever investigation of atmospheric electric phenomena. It can be 1085 accommodated on any short or long duration platform on the Martian surface.

1086 DREAMS is an autonomous instrument suite with an internal power supply unit. It has been designed 1087 to survive and operate in extremely dusty conditions. Its overall mass is $4362 \mathrm{~g}$ including the battery 1088 (1691 g) and the harness. Its power consumption at the nominal battery voltage of $28 \mathrm{~V}$ is $5.60 \mathrm{~W}$ 1089 when in Cruise and Surface states, $7.84 \mathrm{~W}$ during acquisition, $6.72 \mathrm{~W}$ in Upload state and $0.28 \mathrm{~W}$ in 1090 idle state. DREAMS has been fully calibrated and tested in the field. It performed nominally during 1091 the cruise to Mars and was healthy during the descent to Mars. A Spare Model of the instrument, 1092 identical to the one flown on-board Schiaparelli is available for a future mission. 
AC

ADC

AM0

CEU

CFD

COTS

CPU

DC

DREAMS

DSP

EDM

E-field

ESA

FM

FMI

FoV

FPGA

FS

HK

INTA

ISO

LTST

MCU

MEMS

MSL

MTL

NASA

NIR

OBDH

OD

OH
Alternating Current

Analog to Digital Converter

Air Mass 0

Central Electronic Unit

Computational Fluid Dynamics

Commercial-Of-The-Shelf

Central Processing Unit

Direct Current

Dust characterization, Risk assessment and Environment Analyzer on the Martian Surface

Digital Signal Processor

Entry descent and landing Demonstrator Module

Electric field

European Space Agency

Flight Model

Finnish Meteorological Institute

Field of View

Field-Programmable Gate Array

Flight Spare

Housekeeping

Instituto Nacional de Técnica Aeroespacial

International Standards Organization

Local True Solar Time

Microcontroller Unit

Micro Electro-Mechanical System

Mars Science Laboratory

Mission TimeLine

National Aeronautics and Space

Administration

Near Infrared

On-Board Data Handling

Optical Depth

Optical Head 
P1/P2

PCB

PDS4

PE

PTFE

RDS

REF

REMS

RH

RTD

SIS

SNR

SPASOLAB

SSW

SZA

TID

TGO

TVT

UHF

UTC

UV
DREAMS Pressure Sensor 1/2

Printed Circuit Board

Planetary Data System version 4

Processing Electronics

Polytetrafluoroethylene

Radiation and Dust Sensor

Ground reference model

Rover Environmental Monitoring Station

Relative Humidity

Resistance Temperature Detector

Solar Irradiance Sensor

Signal to Noise Ratio

Space Solar Cell Test Laboratory

Sensor SWitch sequence

Solar Zenith Angle

Total ionizing Dose

Trace Gas Orbiter

Thermal Vacuum Test

Ultra High Frequency

Universal Time Coordinated

UltraViolet 


\section{Acknowledgments}

This work was supported by the Italian Space Agency through the agreement I/018/12/0:

“DREAMS EDM Payload ExoMars 2016.” The development of the DREAMS instrument was funded and coordinated by ASI.

DREAMS is the result of a cooperation of six European Countries (Italy, France, Spain,

Netherlands, Finland, United Kingdom) led by Italy. DREAMS is built by UPD-CISAS with contribution from LATMOS/FMI/INTA/Oxford University/INAF-OAC, operated by INAFOAC/UPD-CISAS and provided by ASI.

\section{References}

Álvarez M., C. Hernando, J. J. Jiménez, F. J. Álvarez, I. Martín and D. Escribano, TID results of optical materials and photodiodes for SIS instruments (DREAMS project), in Proc. of IEEE Nuclear and Space Radiation Effects Conference, Paris, France (2014).

Álvarez M., J. J. Jiménez, D. Escribano, P. Manzano, I. Arruego, V. Apéstigue, M. GonzálezGuerrero, Low Dose Rate TID testing of ADXL327 Accelerometer for a Mars Mission, in Proc. of IEEE Nuclear and Space Radiation Effects Conference, Boston, Massachusetts (2015).

Apéstigue V., I. Arruego, J. Martínez, J.J. Jiménez, J. Rivas, M. González, J. Álvarez, J. Azcue, A. Martín-Ortega, J.R. de Mingo, M. T. Álvarez, L. Bastide, A. Carretero, A. Santiago, I. Martín, B. Martín, M.A. Alcacera, J. Manzano, T. Belenger, R. López, D. Escribano, P. Manzano, J. Boland, E. Cordoba, A. Sánchez-Lavega, S. Pérez, A. Sainz López, M. Lemmon, M. Smith, C. E. Newman, J. Gómez Elvira, N. Bridges, P. Conrad (7), M. de la Torre Juarez, R. Urqui, J.A. Rodríguez Manfredi. Radiation and Dust Sensor for MARS2020: technical design and development status overview, in Proc. of European Planetary Science Congress, Vol 10. (2015) 
Aplin K.L., Atmospheric electrification in the Solar System, Surveys in Geophysics, 27, 1, 63-108 (2006). doi: 10.1007/s10712-005-0642-9.

Arruego I., V. Apéstigue, J. Jiménez-Martín, J. Martínez-Oter, F.J. Álvarez-Ríos, M. González-

Guerrero, J. Rivas, J. Azcue, I. Martín, D. Toledo, L. Gómez, M. Jiménez-Michavila, M. Yela, DREAMS-SIS: the Solar Irradiance Sensor on-board the ExoMars 2016 Lander, submitted for publication to Advances in Space Research, (2016).

Guerrero, J. Rivas, J. Azcue, I. Martín, D. Toledo, L. Gómez, M. Jiménez-Michavila, M. Yela, DREAMS-SIS: the Solar Irradiance Sensor on-board the ExoMars 2016 Lander, Advances in Marshall J.R., Rafkin S.C. R., Catling D.C., Oxidant Enhancement in Martian Dust Devils and Storms: Implications for Life and Habitability, Astrobiology, Vol. 6, Iss. 3, pp. 439-450 (2006).

Bertrand T., A. Spiga, S. Rafkin, A. Colaitis, F. Forget, E. Millour, An intercomparison of LargeEddy Simulations of the Martian daytime convective boundary layer, Geosci. Model Dev.

$$
\text { Discuss., doi:10.5194/gmd-2016-241 (2016). }
$$

Berthelier, J.., Grard, R., Laakso, H., Parrot, M., ARES, atmospheric relaxation and electric field sensor, the electric field experiment on NETLANDER. Planetary and Space Science 48, 11931200 (2000). doi:10.1016/S0032-0633(00)00103-3

Chiodini S., G. Colombatti, M. Pertile, S. Debei, Numerical study of lander effects on DREAMS scientific package measurements, IEEE Metrology for Aerospace (MetroAeroSpace), 433-438 (2014).

Chiodini S., G. Colombatti, E. Friso, M. Pertile, S. Debei, “Multiphysics modelling of MarsTEM shield,” in 2015 IEEE Metrology for Aerospace (MetroAeroSpace), pp. 271- 276 (2015). 
1146 Chojnacki M., Urso A., Fenton L.K., Michaels T.I., Aeolian dune sediment flux heterogeneity in 1147 Meridiani Planum, Mars, Aeolian Research (2016) in press.

1148 Colombatti G., S. Chiodini, E. Friso, A. Aboudan, C. Bettanini, S. Debei, F. Esposito, "MarsTEM: 1149 The temperature sensor of the DREAMS package onboard Exomars2016,” in 2014 IEEE 1150 Metrology for Aerospace (MetroAeroSpace), pp. 249-254 (2014).

1151 Colombatti G., S. Chiodini, E. Friso, A. Aboudan, C. Bettanini, M. Poli, S. Debei, F. Esposito, C. 1152 Molfese, P. Schipani, R. Mugnuolo, S. Pirrotta, E. Marchetti, MARSTEM FIELD TEST IN 1153 MARS ANALOG ENVIRONMENT, in 2015 IEEE Metrology for Aerospace (MetroAeroSpace, 1154 2015).

1155 Déprez G., Micro-ARES on ExoMars 2016, PhD thesis, to be published (2016).

1156 Esposito F., R. Molinaro, C. I. Popa, C. Molfese, F. Cozzolino, L. Marty, K. Taj-Eddine, G. Di 1157 Achille, G. Franzese, S. Silvestro, and G. G. Ori,, The role of the atmospheric electric field in the 1158 dust-lifting process, Geophys. Res. Lett., 43 (2016). doi:10.1002/2016GL068463.

1159 Farrell, W.M., McLain, J.L., Collier, M.R., Keller, J.W., Jackson, T.J., Delory, G.T., Is the electron 1160 avalanche process in a martian dust devil self-quenching? Icarus 254, 333-337 (2015).

1161 doi:10.1016/j.icarus.2015.04.003

1162 Formisano, V., Atreya, S., Encrenaz, T., Ignatiev, N., Giuranna, M., Detection of Methane in the 1163 Atmosphere of Mars, Science, 306, Iss. 5702, pp. 1758-1761 (2004).

1164 Harri, A.-M., M. Genzer, O. Kemppinen, H. Kahnapää, J. Gomez-Elvira, J.A. Rodriguez-Manfredi, 1165 R. Haberle, J. Polkko, W. Schmidt, H. Savijärvi, J. Kauhanen, E. Atlaskin, M. Richardson, T. 1166 Siili, M. Paton, M. de La TorreJuarez, C. Newman, S. Rafkin, M.T. Lemmon, M. Mischna, S. 1167 Merikallio, H. Haukka, J. Martin-Torres, M.-P. Zorzano, V. Peinado, R. Urqui, A. Lepinette, A. 1168 Scodary, T. Mäkinen, L. Vazquez, N. Rennó, and the REMS/MSL Science Team, Pressure 1169 observations by the Curiosity rover - Initial results, J. Geophys. Res. 119, 82-92 (2014a). 1170 Harri, A.-M., M. Genzer, O. Kemppinen, J. Gomez-Elvira, R. Haberle, J. Polkko, H. Savijärvi, N. 1171 Rennó, J.A. Rodriguez-Manfredi, W. Schmidt, M. Richardson, T. Siili, M. Paton, M. de la Torre- 
Juarez, T. Mäkinen, C. Newman, S. Rafkin, M. Mischna, S. Merikallio, H. Haukka, J. MartinTorres, M. Komu, M.-P. Zorzano, V. Peinado, L. Vazquez, and R. Urqui, Mars Science Laboratory Relative Humidity Observations - Initial results, J. Geophys. Res. 119, 2132-2147 (2014b).

Harrison R.G., E. Barth, F. Esposito, J. Merrison, F. Montmessin, K.L. Aplin, C. Borlina, J.J. Berthelier, G. Déprez, W. Farell, I.M.P. Houghton, N.O. Renno, K.A. Nicoll, S.N. Tripathi, M. Zimmerman, Applications of electrified dust and dust devil electrodynamics to Martian atmospheric electricity, Space Science Review (2016), doi: 10.1007/s11214-016-0241-8.

Holstein-Rathlou C., J. Merrison, J. J. Iversen, A. B. Jakobsen, R. Nicolajsen, P. Nørnberg, K.

Rasmussen, A. Merlone, G. Lopardo, T. Hudson, D. Banfield, and G. Portyankina, An

Environmental Wind Tunnel Facility for Testing Meteorological Sensor Systems, Journal of Atmospheric and Oceanic Technology, vol. 31, issue 2, pp. 447-457 (2014).

Jimenez J.J., Oter J.M., Apestigue V., Hernando C., Ibarmia S., Hajdas W., Sanchez-Paramo J., Alvarez M.T., Arruego I., Guerrero H., Proton Monitor Las Dos Torres: First Intercomparison of In-Orbit Results, IEEE Transactions on Nuclears Science, Vol. 59, No. 4 (2012).

Jiménez J. J., F.J Álvarez, M. Gonzalez-Guerrero, V. Apéstigue, I. Martín, J. M Fernández, A. A. Fernán, I.Arruego, Calibration OGSE for a Multichannel Radiometer for Mars Atmosphere Studies, in Proc. of International Conference on Space Optics, ICSO, Biarritz, France (2016).

Mumma, M.J., Villanueva, G.L., Novak, R.E., Hewagama, T., Bonev, B.P., DiSanti, M.A., Mandell, A.M., Smith, M.D., Strong Release of Methane on Mars in Northern Summer 2003, Science, Volume 323, Issue 5917, pp. 1041- (2009).

Murphy J., K. Steakley, M. Balme, G. Deprez, F. Esposito, H. Kahanpää, M. Lemmon, R. Lorenz, N. Murdoch, L. Neakrase, M. Patel, P. Whelley, Field Measurements of Terrestrial and Martian Dust Devils, Space Science Reviews (2016). Doi: 10.1007/s11214-016-0283-y

Nikkanen T., Schmidt W., Harri A.-M., Genzer M., Hieta M., Haukka H., Kemppinen O., Space 
qualification of an automotive microcontroller for the DREAMS-P/H pressure and humidity instrument on board the ExoMars 2016 Schiaparelli lander, EPCS2015-465 (2015).

Schipani, P., Marty, L., Mannetta, M., Esposito, F., Molfese, C., Aboudan, A., Apestigue-Palacio, V., Arruego-Rodríguez, I., Bettanini, C., Colombatti, G., Debei, S., Genzer, M., Harri, A.-M., Marchetti, E., Montmessin, F., Mugnuolo, R., Pirrotta, S., Wilson, C., The ExoMars DREAMS scientific data archive, Proc. SPIE 9913, 99134F (2016).

Seiff A., Tillman J.E., Murphy, J.R., Schofield J.T., Crisp, D., Barnes, J.R., LaBaw C., Mahoney C., Mihalov J.D., Wilson G.R., Haberle R., The atmosphere structure and meteorology instrument on the Mars Pathfinder lander, J. Geophys. Res., 102, Iss. E2, p. 4045-4056 (1997).

Silvestro S., Vaz D.A., Fenton L.K., Geissler P.E., Acive aeolian processes on Mars: A regional study in Arabia and Meridiani Terrae, Geophysical Research Letters, L20201 (2011). doi:10.1029/2011GL048955.

Silvestro S., Vaz D.A., Di Achille G., Popa I.C., Esposito F., Evidence for different episodes of aeolian construction and a new type of wind streak in the 2016 ESA ExoMars landing ellipse in Meridiani Planum, Mars, Journal of Geophysical Research: Planets, 120, Iss. 4, pp. 760-774 (2015). doi:10.1002/2014JE004756.

Smith M.D., María-Paz Zorzano, Mark Lemmon, Javier Martín-Torres, Teresa Mendaza de Cal, Aerosol optical depth as observed by the Mars Science Laboratory REMS UV photodiodes, Icarus 280, 234-248 (2016).

D. Toledo, I. Arruego, V. Apéstigue, J.J. Jiménez, L. Gómez, M. Yela, P. Rannou, J.-P. Pommereau, “Measurement of dust optical depth using the solar irradiance sensor (SIS) onboard the ExoMars 2016 EDM”, Planetary and Space Science 138 (2017) 33-43.

Towner, M. C., Patel, M. R., Ringrose, T. J., Zarnecki, J. C., Pullan, D., Sims, M. R., Haapanala, S., Harri, A. M., Polkko, J., Wilson, C. F., Zent, A. P., Quinn, R. C., Grunthaner, F. J., Hecht, M. H., Garry, J. R. C., The Beagle 2 environmental sensors: science goals and instrument description. Plan. Space Sci., 52: 1141-1156 (2004). 
1223 Vago, J., Witasse, O., Svedhem, H., Baglioni, P., Haldemann, A., Gianfiglio, G., Blancquaert, T., 1224 McCoy, D., de Groot, R., ESA ExoMars program: The next step in exploring Mars, Solar 1225 System Research, Volume 49, Issue 7, pp.518-528 (2015).

1226 Vidali I., MarsTEM, un termometro per la misura della temperatura atmosferica marziana: 1227 progettazione, prototipazione e studio degli effetti dovuti all'autoriscaldamento, Bachalor Thesis, 1228 Padova Univ. (2012).

1229 Webster C.R., P.R. Mahaffy, S.K. Atreya, G.J. Flesch, M.A. Mischna, P.-Y. Meslin, K.A. Farley, 1230 P.G. Conrad, L.E. Christensen, A.A. Pavlov, J.Martín-Torres, M.-P. Zorzano, T.H. McConnochie, 1231 T. Owen, J.L. Eigenbrode, D.P. Glavin, A. Steele, C.A. Malespin, P. D. Archer Jr. B. Sutter, P. 1232 Coll, C. Freissinet, C.P. McKay, J.E. Moores, S.P. Schwenzer, J.C. Bridges, R. Navarro-Gonzalez, 1233 R. Gellert, M.T. Lemmon, the MSL Science Team, Mars methane detection and variability at Gale 1234 crater, Science, 347, Iss. 6220, pp. 415-417 (2015).

1235 Wilson C. F., Calcutt S. B., Jones T. V., The Beagle 2 wind sensor, EGS - AGU - EUG Joint 1236 Assembly, abstract \#691 (2003).

1237 C.F. Wilson, Measurement of wind on the surface of Mars, DPhil thesis, 2003b.

1238 Wilson C.F., Camilletti A. L., Calcutt S. B., Ligrani P. M., A wind tunnel for the calibration of 1239 Mars wind sensors, Planet. Space Sci., 56, Iss. 11, p. 1532-1541 (2008). doi:10.1016/j.pss.2008.05.011 